\title{
烯醇类化合物氧化偶联反应研究进展
}

\author{
陈伟 $a, b$ 刘 强*, $a$ \\ $\left({ }^{a}\right.$ 南方医科大学中医药学院 广州 510515) \\ ( $b$ 粤澳中医药科技产业园开发有限公司 广东珠海 519031)
}

\begin{abstract}
摘要 烯醇类化合物氧化偶联反应作为直接构建 $\mathrm{C}-\mathrm{C}$ 键的高效合成方法之一，是有机化学领域研究关注的重点. 尽 管首例烯醇类化合物氧化偶联反应早在 1935 年就已被报道, 但直到 20 世纪 70 年代才得到化学家们的广泛重视, 不过 当时反应的有效性和实用性仍有待进一步提高. 近年来, 该反应在有机合成方法学和天然产物全合成方面都取得了快 速的发展. 因此, 重点概述了近十年来该类型反应合成方法学的发展及其在天然产物全合成的应用研究进展.

关键词 氧化偶联反应; 烯醇类化合物; 碳碳键; 方法学; 天然产物
\end{abstract}

\section{Recent Advances in the Oxidative Coupling Reaction of Enol Derivatives}

\author{
Chen, Wei ${ }^{a, b} \quad$ Liu, Qiang*,a \\ ( ${ }^{a}$ School of Chinese Medicine, Southern Medicinal University, Guangzhou 510515) \\ ( ${ }^{b}$ Guangdong-Macau Traditional Chinese Medicine Technology Industrial Park Development Co., Ltd., \\ Zhuhai, Guangdong 519031)
}

\begin{abstract}
Oxidative coupling reaction of enol derivatives (enolates, enol silanes, enamines, etc.) provides an efficient method for the direct construction of carbon-carbon bond, which plays a prominent role in the field of synthetic chemistry. Although the first case of this reaction had been reported in 1935, it was not until the 1970s that chemists began to pay more attention to this reaction. However, the effectiveness and practicability of this reaction were still somewhat limited at that time. In recent years, this reaction has been developed rapidly in the field of synthetic methodology and the total synthesis of natural products. Therefore, these advances in methodologies and its applications in the total synthesis of natural products in the last decades are mainly summarized.
\end{abstract}

Keywords oxidative coupling reaction; enol derivatives; carbon-carbon bond; methodology; natural product

偶联反应作为一类在 20 世纪 60 年代被有机化学家 开发出来用于 $\mathrm{C}-\mathrm{C}$ 键形成的新型反应之一 (Scheme 1). 从发现之初至今, 该反应在底物普适性和官能团容忍性 等方面表现出独特的优势, 成为有机化学研究领域的热 点之一, 同时也被广泛应用于生物医药、农药、多功能 材料等众多领域, 极大地促进了有机化学的发展 ${ }^{[1]}$. 与 经典的偶联反应相比, 氧化偶联反应不仅减少了纷繁复 杂的官能团转化操作, 提高了合成效率, 从而实现反应 的步骤经济性, 更符合当今绿色化学的发展要求 ${ }^{[2]}$. 因 此, 氧化偶联反应也被誉为 “化学中的圣杯(Holy Grails in Chemistry)” [3].

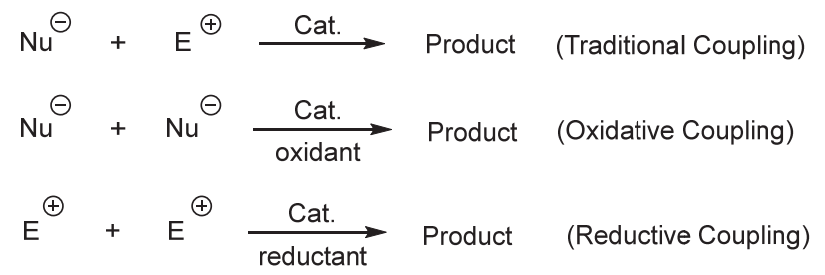

图式 1 偶联反应的三种形式

Scheme 1 Three types of the coupling reaction

烯醇类化合物氧化偶联反应作为发生在两个亲核 试剂之间的反应，无需预先对底物进行官能团化即可一 步得到 1,4-二羰基骨架, 是一种直接形成 $\mathrm{C}-\mathrm{C}$ 键的高

\footnotetext{
* Corresponding author. E-mail: gzlq2002@163.com

Received April 28, 2021; revised May 10, 2021; published online June 15, 2021.

Project supported by the China Postdoctoral Science Foundation (No. 2020M682925).

中国博士后科学基金(No. 2020M682925)资助项目.
} 
效合成方法(Scheme 2) ${ }^{[4]} .1935$ 年, Ivanoff 和 Spassoff 等 ${ }^{[5]}$ 报道了首例烯醇类化合物自身氧化偶联反应，不过 由于该反应副产物多、产率低, 从而限制了其在有机合 成中的应用，这也使得该领域的研究在此后数十年间一 直处于停滞状态. 从 20 世纪 70 年代开始, 有机化学家 重新对该反应进行了全面的研究, 包括底物的扩展和应 用 ${ }^{[6]}$ 、氧化剂的选择 ${ }^{[7]}$ 、反应的类型 ${ }^{[8]}$ 、反应的机理 ${ }^{[9]}$ 和反应的立体选择性 ${ }^{[10]}$ 等方面, 使得该反应的普适性 和有效性不断提升, 极大地促进了该反应的发展. 无其 是 2005 年以来, Baran 等 ${ }^{[11]}$ 对烯醇类化合物交叉氧化偶 联反应进行了深入的研究, 并将该反应成功应用于多个 复杂天然产物的全合成中, 极大地提高了合成效率, 证 明该类型反应在天然产物全合成中具有广阔的应用前 景.
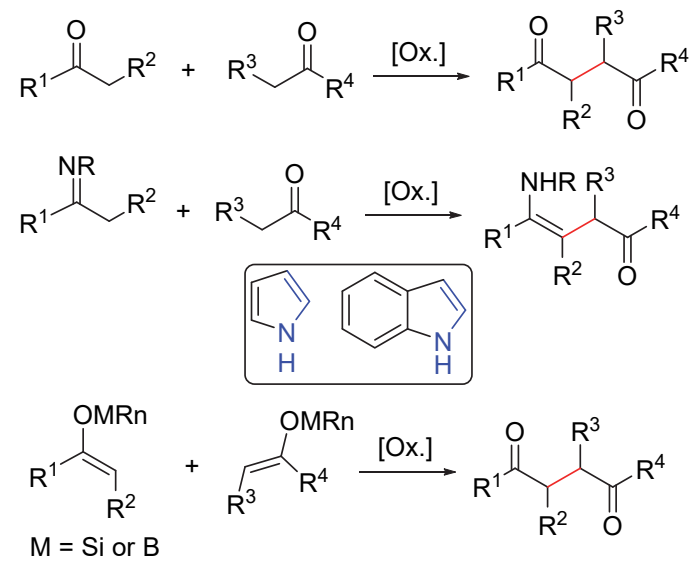<smiles>[R]C=C([R17])O[R]([R])([R])O/C([R])=C\[R]</smiles>

$$
\stackrel{[\mathrm{Ox} .]}{\longrightarrow}
$$

$\mathrm{M}=\mathrm{Si}$ or $\mathrm{Ti}$ or Z

图式 2 烯醇类化合物氧化偶联反应的类型

Scheme 2 Oxidative coupling reaction of enol derivatives

自 1935 年发现第一例烯醇类化合物氧化偶联反应 至今八十多年间, 在一代又一代有机化学家的不解努力 下, 该类型反应已经实现了数次飞跃式的发展, 分别体 现在反应的选择性、有效性、普适性和实用性等方 面 ${ }^{[6-10]}$. 对于该领域 2010 年前大多数的研究工作, Plumet $^{[12]} 、 \operatorname{Baran}^{[13]} 、$ Dong $^{[14]} 、$ Thomson $^{[15]}$ 、马大为 ${ }^{[16]}$ 等已进行了相应的总结. 因此, 本综述着重概述了从 2010 年至今近十年来烯醇类化合物氧化偶联反应的最 新研究进展, 内容包括该反应在有机合成方法学方面的 发展及其在天然产物全合成方面的应用, 以期为关注此 领域的有机化学家提供更多有益的启发, 同时也为推动 该类型反应未来的进一步发展提供更多的参考.

\section{1 烯醇类化合物氧化偶联反应方法学的发展}

\section{1 经典烯醇形式底物的氧化偶联反应}

经典烯醇形式底物的氧化偶联反应是指羰基化合 物(包括酮、羧酸、酯、酰胺等)在一定条件下首先形成 烯醇(enols)或其碱金属的盐(enolates), 然后在单电子氧 化剂的作用下得到羰基 $\alpha$ 位自由基，之后通过偶联反应 实现相应 $\mathrm{C}-\mathrm{C}$ 键的构建. 早期的烯醇类化合物氧化偶 联反应基本都是该类型底物的反应. 由于不同烯醇形式 底物之间的氧化偶联反应往往存在较多的竞争反应，如 自身氧化偶联反应、Claisen 缩合反应 ${ }^{[17]}$, 如何实现不同 分子间化学选择性的交叉氧化偶联反应一直是该领域 研究的热点和难点之一. 1975 年, Saegusa 和 Itoh 两个研 究小组 ${ }^{[18]}$ 几乎同时报道了不同烯醇形式底物之间的交 叉氧化偶联反应，其主要合成策略是使用过量的一分子 烯醇形式化合物. 2005 2008 年, Baran 研究小组真正实 现了物质的量比为 $1: 1$ 下不同烯醇形式底物间的交叉 氧化偶联反应(Scheme 3) ${ }^{[11]}$, 并将该反应成功应用于天 然产物(一)-bursehernin 的不对称全合成中 ${ }^{[19]}$.

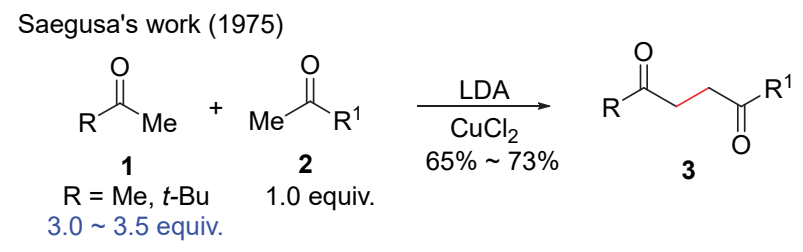

Itoh's work (1975)

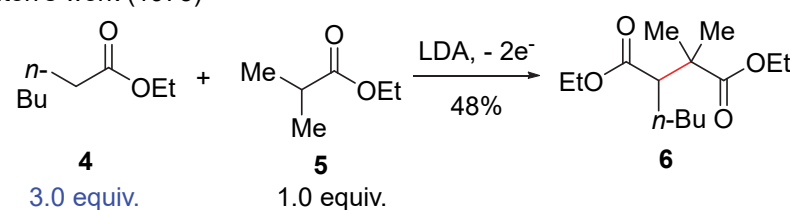

Baran's work (2005 2008)

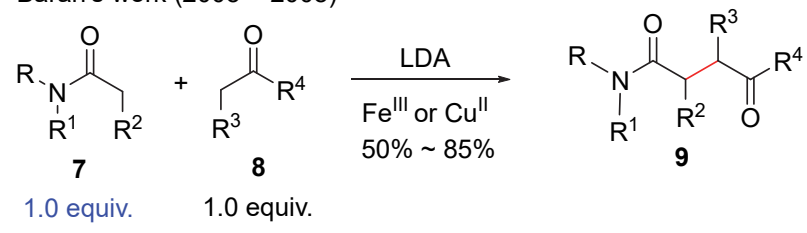

图式 3 早期报道的烯醇形式底物交叉氧化偶联反应

Scheme 3 Oxidative cross-coupling reaction of enolates

2011 年, Flowers 以物质的量比为 $1: 1$ 的酮类底物 烯醇锂盐 $\mathbf{A}$ 和 $\mathbf{B}$ 进行分子间交叉氧化偶联反应，以中等 到较好的收率得到了相应的交叉偶联产物 $\mathbf{C}(\text { 表 1 })^{[20]}$. 更重要的是，他们通过使用 ${ }^{7} \mathrm{Li} \mathrm{NMR}$ 光谱分析发现了底 物的烯醇锂盐在反应溶剂中主要以交叉聚集的形式存 在 $\left(\mathrm{A}_{2} \mathrm{~B}_{2}\right)$, 而非自身二聚体的形式 $\left(\mathrm{A}_{4}+\mathrm{B}_{4}\right)$, 且最终偶 联产物的比例与两者的比例 $\left(\mathrm{A}_{2} \mathrm{~B}_{2} / \mathrm{A}_{4}+\mathrm{B}_{4}\right)$ 呈现正相关 $\left(R^{2}=0.999\right)$, 提示可以通过调控碱金属烯醇盐在溶剂体 
表 1 Flower 研究小组报道的烯醇锂盐聚集效应对交叉氧化偶联反应的影响 ${ }^{a}$

Table 1 Flowers' studies on Li-enolate aggregation effects of oxidative cross-coupling reaction<smiles>[R]c1ccc2c(c1)CCC=C2O</smiles>

A

1.0 equiv.

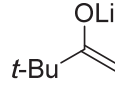

B

1.0 equiv.<smiles>[R][CH]C(C)(C)C(=O)CC1CCc2ccccc2C1=O</smiles>

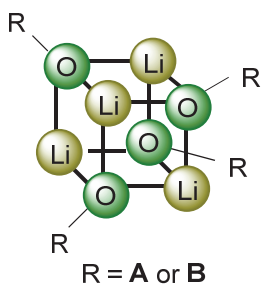

\begin{tabular}{|c|c|c|c|c|c|}
\hline Ketone A & Ketone B & Product $\mathbf{C}$ & $\mathrm{A}_{2} \mathrm{~B}_{2} / \mathrm{A}_{4}+\mathrm{B}_{4}$ & Product ratio & Yield/\% \\
\hline & & & $15.7: 1$ & $13.8: 1$ & 62 \\
\hline & & & $14.7: 1$ & $12.8: 1$ & 58 \\
\hline & & & $14.3: 1$ & $12.4: 1$ & 62 \\
\hline & & & $8.5: 1$ & $7.0: 1$ & 46 \\
\hline & & & 4.1:1 & $3.0: 1$ & 47 \\
\hline
\end{tabular}

${ }^{a}$ Ratios and yields determined by NMR spectroscopy. $\mathrm{CAN}=$ ceric ammonium nitrate.

Kise's work (1995)

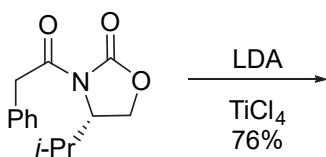

10 $76 \%$

(n)<smiles>CCC[C@@H]1COC(=O)N1C(=O)C(c1ccccc1)C(C(=O)N1C(=O)OC[C@H]1P)c1ccccc1</smiles>

11

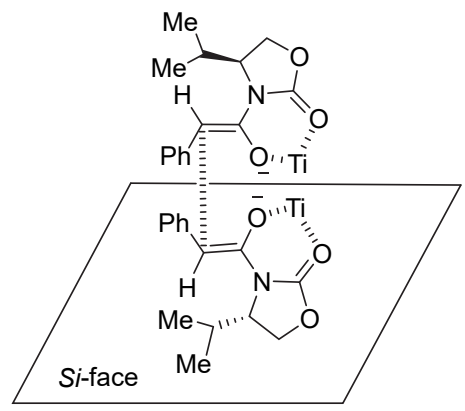

Schäfer's work (2001)<smiles>O=C(Cc1ccccc1)N1CCOC1=O</smiles><smiles>O=C1OCCN1C(=O)C(c1ccccc1)C(C(=O)N1CCOC1=O)c1ccccc1</smiles>

13

dl:meso $=25: 75$

$76 \%$ ee

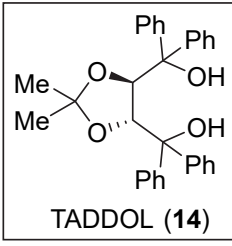

图式 4 早期报道的立体选择性自身氧化偶联反应

Scheme 4 Enantioselective oxidative homo-coupling reaction of titanium enolates

系中的存在形式来实现化学选择性的交叉氧化偶联反 应.
随着对烯醇类化合物氧化偶联反应研究的不断深 入, 有机化学家开始把目光转向对该反应立体选择性的 
探究, 以获得更为有用的光学纯 1,4-二羰基化合物. 1995 年, Kise 等 ${ }^{[10]}$ 报道了使用 Evans 手性恶唑烷酮 10 来实现立体选择性的自身氧化偶联反应, 以 $76 \%$ 的收率 得到 2,3-取代的琥珀酸衍生物对映体 11. 不过, 由于该 反应手性辅基的引入和脱除需要几步的转化, 在步骤经 济性方面略显不足. 2001 年, Schäfer ${ }^{[21]}$ 通过使用手性配 体 TADDOL (14)和四氯化钛成功实现了该类型反应的 不对称形式, 虽然反应最优的对映选择性 $(76 \% e e)$ 和非 对映选择性 $(d l: m e s o=25: 75)$ 偏低 (Scheme 4), 但是 该研究工作为后续烯醇类化合物不对称形式氧化偶联 反应相关研究提供了很好的思路和参考.

此外, 直接通过手性底物来实现立体选择性的烯醇 类化合物氧化偶联反应同样被证明是一种行之有效的 合成方法. 2011 年, Thomson 等 ${ }^{[22]}$ 从光学纯的底物 $\mathbf{1 5}$ (99: 1 er) 出发, 在经典的二异丙基氨基锂 $(\mathrm{LDA}) / \mathrm{CuCl}_{2}$ 反应条件下以 $66 \%$ 的收率顺利得到单一的自身氧化偶 联产物 16, 随后在路易斯酸加热的条件下进行芳构化 得到具有轴手性的联苯酚 17 (Scheme 5), 并最终实现了 咔唑类天然产物 bismurrayaquinone A 的不对称全合成.
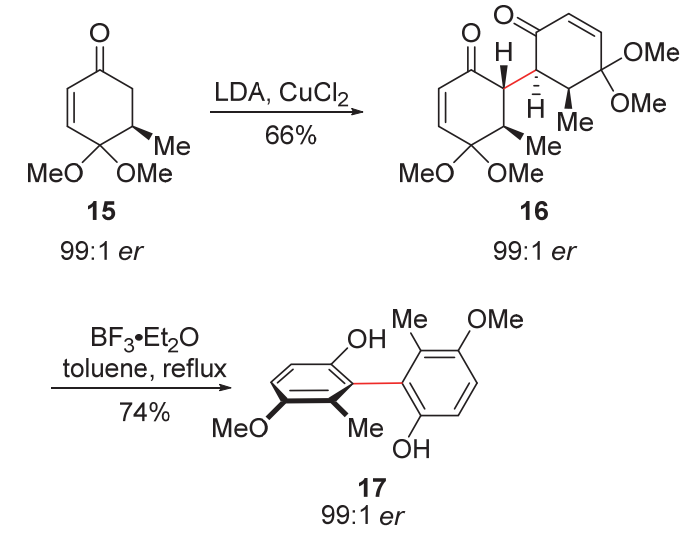

图式 5 利用自身氧化偶联反应合成轴手性联苯酚 Scheme 5 Enantioselective synthesis of biphenol via oxidative homo-coupling reaction reported by Thomson's group

虽然有机化学家已经发展出烯醇类化合物自身氧 化偶联反应的不对称形式, 但烯醇类化合物对映选择性 的交叉氧化偶联反应依然是该领域研究亟待解决的一 大挑战. 2018 年, Alezra 等 ${ }^{[23]}$ 报道了一种通过手性底物 控制的不对称形式交叉氧化偶联反应. 他们巧妙地利用 光学纯恶唑烷酮底物 18 的 “手性记忆(memory of chirality)” 效应, 以优异的对映选择性(最高可达 $94 \% e e$ ) 成功合成具有全碳季碳手性中心的 $\alpha$-氨基酸衍生物 20 . 具体而言, 首先在双(三甲基硅基)氨基钾(KHMDS)存在 的条件下, 通过动态动力学拆分对优势构象底物 18 $(P, c i s)$ 进行去质子化得到具有轴手性的烯醇钾盐中间体 I, 随后在单电子氧化剂铜盐的作用下形成自由基中间
体 II, 烯醇化合物 19 从荎基立体位阻较小的背面进攻 生成具有手性保持的全碳季碳立体中心目标产物 20 (Scheme 6).

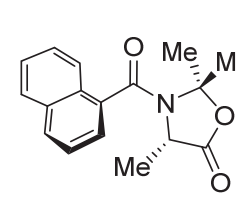

18

$(P$, cis $)$

1.0 equiv.

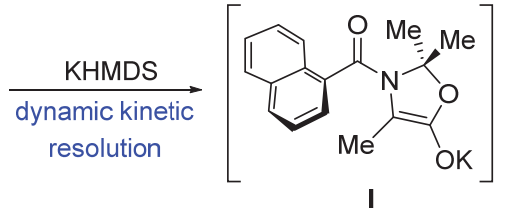

enolate with axial chirality
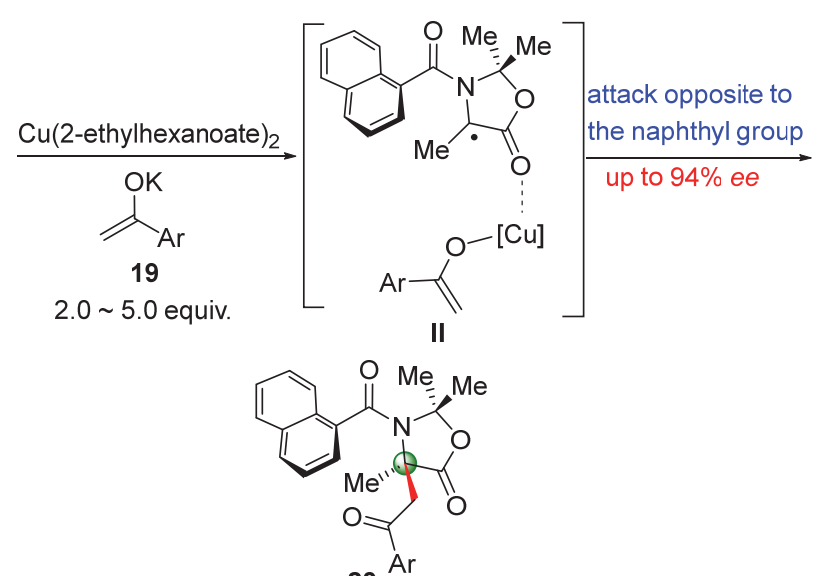

$20 \mathrm{Ar}$

retention of configuration

图式 6 首例对映选择性的交叉氧化偶联反应 Scheme 6 First enantioselective oxidative cross-coupling reaction of enolates reported by Alezra's group

除了对映选择性的交叉氧化偶联反应之外，该类型 反应氧化剂用量的问题也是未来需要进一步突破的瓶 颈问题. 前面所列举的例子全都需要使用化学计量比的 单电子氧化剂(不含电解法). 因此, 能否实现催化量氧 化剂的使用, 是该类型反应未来发展的重点方向之一, 同时也是新时代绿色化学的发展要求. 2012 年, Daugulis 等 ${ }^{[24]}$ 报道了利用催化量 $(10 \mathrm{~mol} \%)$ 的铜盐作单电子氧化 剂, 以氧气作终氧化剂, 并通过添加亲电性的锌盐来稳 定烯醇负离子反应中间体, 成功实现了催化形式下的烯 醇化合物自身氧化偶联反应. 尤值一提的是，在金属物 种以及氧气存在的条件下, 21 的烯醇锂盐十分容易生成 羰基 $\alpha$ 位羟基化的产物 23, 但该反应依然能以中等到较 好的收率得到所需的偶联产物 22 (Scheme 7) ${ }^{[25]}$, 说明该 反应具有良好的化学选择性和底物适用性.

此外, 烯醇类化合物氧化偶联反应所得产物 1,4-二 羰基化合物是呋喃、噻吩和吡咯重要的合成前体. 2014 年, 王永强课题组 ${ }^{[26]}$ 报道了铜盐催化下的烯醇形式化 合物自身氧化偶联反应 $\left(\mathrm{Ag}_{2} \mathrm{O}\right.$ 用于促进 $\mathrm{Cu}^{\mathrm{I}}$ 氧化成 $\left.\mathrm{Cu}^{\mathrm{II}}\right)$, 并串联 Paal-Knorr 反应实现 “一锅法” 合成多取代呋喃 (Scheme $8 \mathrm{a})^{[27]}$. 可见, 该反应可以避免化学计量比强碱 


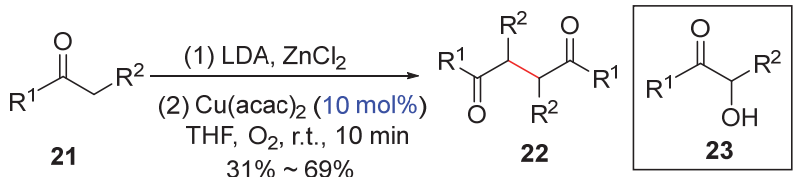

图式 7 铜(II)催化下烯醇类化合物自身化偶联反应

Scheme 7 Copper-catalyzed oxidative homo-coupling reaction of enolates reported by Daugulis's group

(如 LDA、KHMDS 等)的使用, 而直接实现羰基 $\alpha$ 位碳 碳键的构建. 2015 年, 他们 ${ }^{[28]}$ 更是在上述研究工作的基 础上发展出仅使用催化量银盐就能实现一系列多取代 呋喃、噻吩和吡咯的高效合成方法(Scheme 8b).

a: Wang (2014)

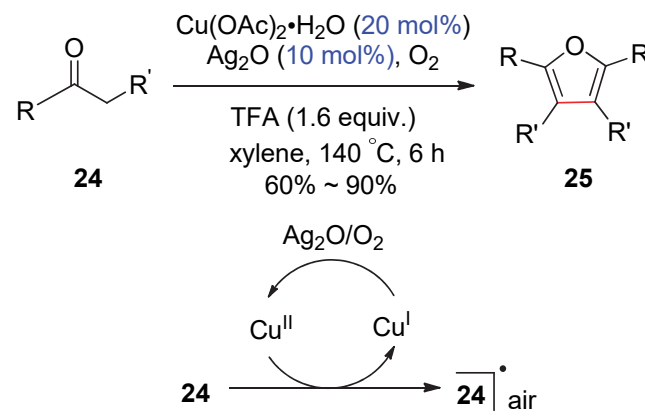

b: Wang (2015) ${ }^{a}$
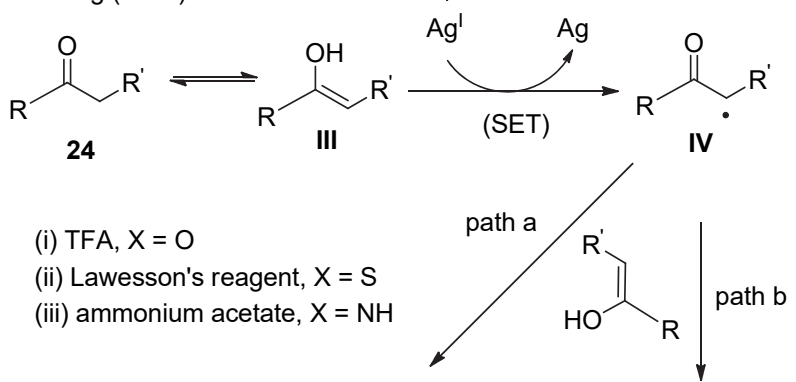

(i) TFA, $X=O$

(ii) Lawesson's reagent, $X=S$

(iii) ammonium acetate, $\mathrm{X}=\mathrm{NH}$

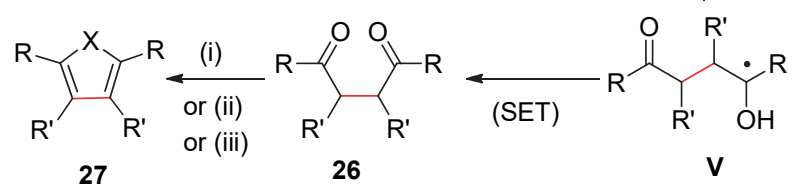

Reaction conditions: AgF (10 mol\%), air, xylene, $140{ }^{\circ} \mathrm{C}, 4 \sim 10 \mathrm{~h}$; SET (single electron transfer)

图式 8 铜(II)或银(I)催化下多取代五元杂环的合成 Scheme 8 Copper or silver-catalyzed syntheses of tetrasubstituted furans, thiophenes and pyrroles reported by Wang's group

相比之下，目前对于催化形式下烯醇类化合物交叉 氧化偶联反应的研究报道则相对较少. 2016 年, Christoffers 等 ${ }^{[29]}$ 报道了 $\beta$-羰基酯 28 和烯醇乙酸酯 $\mathbf{2 9}$ 在催化 量 $(2.5 \mathrm{~mol} \%)$ 三氯化铈水合物单电子氧化剂的作用下成 功实现了交叉氧化偶联反应(Scheme 9).

2018 年, Ohshima 和 Takashi 等 ${ }^{[30]}$ 报道了铁盐催化下 烯醇形式化合物化学选择性的交叉氧化偶联反应. 他们

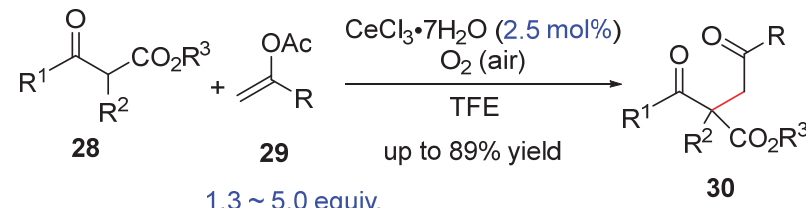

图式 9 铈(III)催化下烯醇类化合物交叉氧化偶联反应

Scheme 9 Cerium catalyzed oxidative cross-coupling reaction of enolates reported by Christoffers's group

通过采用反应过程中形成瞬时二聚体 34 (transient homo-coupling dimer)的合成策略，以催化量 $(10 \mathrm{~mol} \%$ )的 铁盐作单电子氧化剂，二叔丁基过氧化物(DTBP)作终 氧化剂, 成功得到一系列具有相邻连续季碳中心的非天 然 $\alpha, \alpha$-双取代氨基酸衍生物 33 (Scheme 10). 不过，需要 指出的是, 该反应的非对映选择性相对较低(大多数底 物的 $d r$ 值小于 $5: 1$ ), 仍有进一步提升的空间.

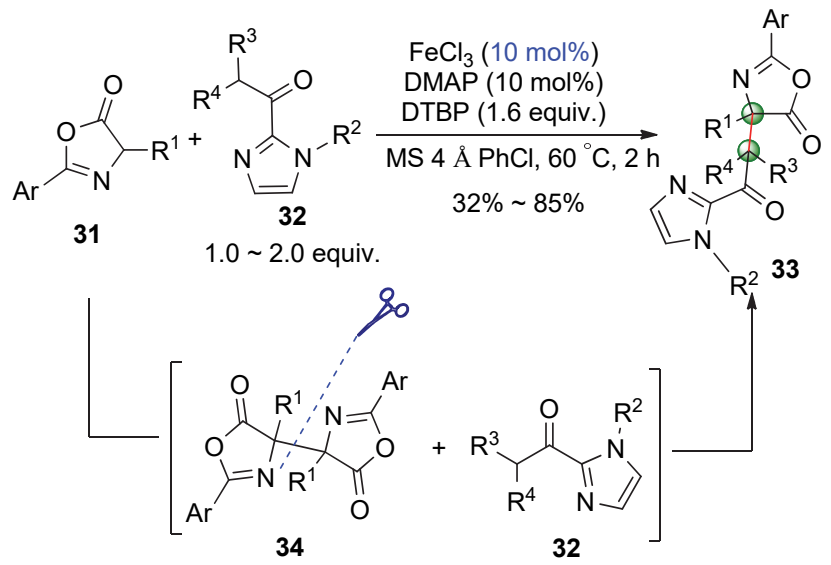

图式 10 铁(III)催化下烯醇类化合物交叉氧化偶联反应 Scheme 10 Iron-catalyzed catalyzed oxidative cross-coupling reaction of enolates reported by Ohshima and Takashi's group

有机电化学合成法在电极上就能实现相关氧化还 原过程, 可以避免化学计量比单电子氧化剂和还原剂的 使用，更符合绿色化学可持续发展的要求，是近些年有 机化学家研究关注的热点领域之一[31]. 虽然早在 1975 年, Itoh 等[18b]便利用有机电合成法成功实现了首例烯醇 形式化合物交叉氧化偶联反应，但直到 2019 年，Meggers 等[32]才报道了利用有机电化学合成方法来实现催 化形式下烯醇类化合物立体专一性的交叉氧化偶联反 应. 该反应的底物适用性广 (38 examples)、产率高(up to 91\%)、对映选择好(up to $99 \%$ ee), 并且还可以构建全碳 季碳手性中心. 其具体的反应过程如下：消旋底物 35 首 先与手性铑催化剂通过配位作用形成复合物中间体 VI, 之后在碱的作用下去质子化生成烯醇化合物中间体 VII，随后进行阳极化学选择性的氧化反应生成自由基 中间体 VIII 并被体系中富电子的烯醇硅醚底物 36 捕获 
形成自由基中间体 $\mathbf{I X}$ 中的 $\mathrm{C}-\mathrm{C}$ 键, 然后中间体 $\mathbf{I X}$ 在 阳极进行单电子氧化得到中间体 $\mathbf{X}$, 最后脱去硅基正离 子和进行产物/底物的交换完成整个催化循环(Scheme 11).
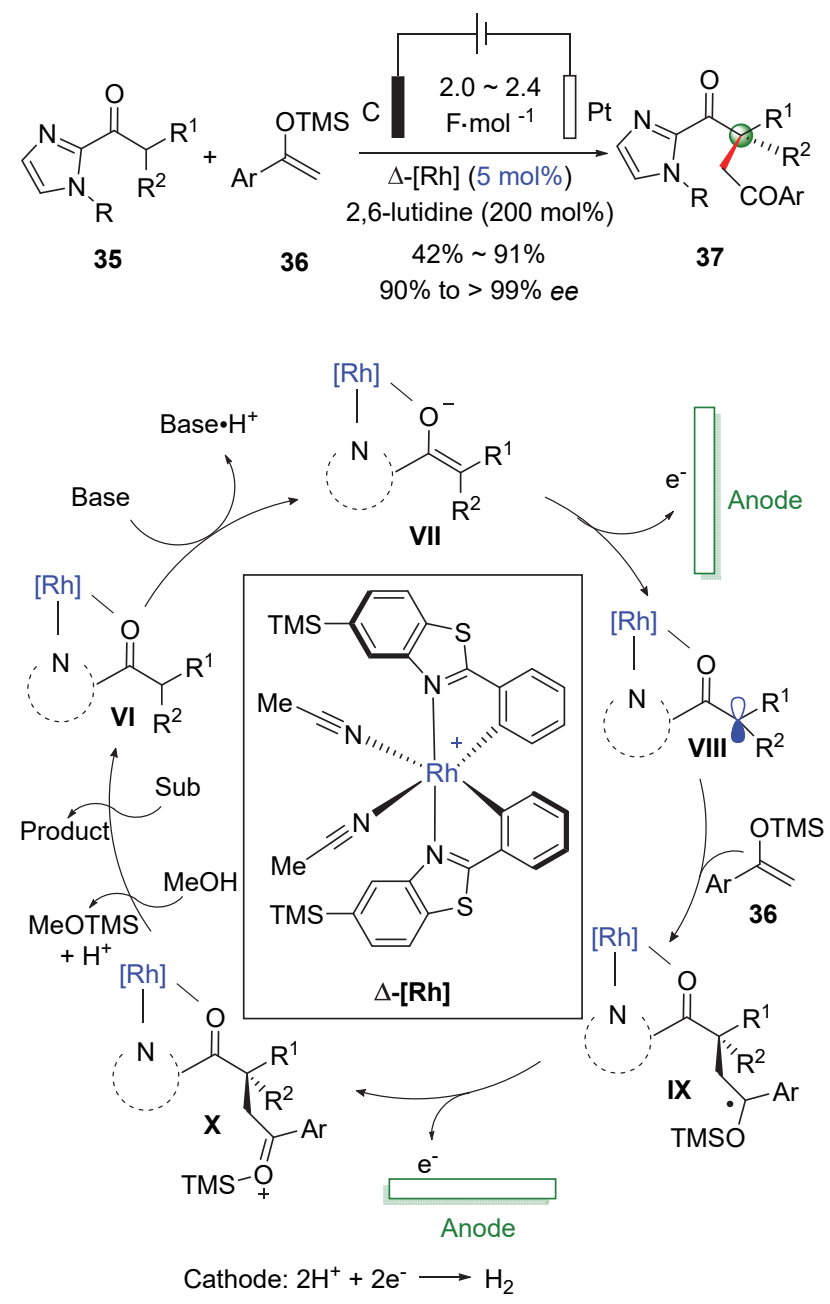

图式 11 催化形式下烯醇类化合物不对称电化学合成 Scheme 11 Catalytic asymmetric electrosynthesis of enantioenriched molecules from enolates reported by Meggers's group

\section{2 烯醇硅醚的氧化偶联反应}

1989 年, Ruzziconi 等 ${ }^{[33]}$ 报道了烯醇硅醚分子间的 交叉氧化偶联反应，他们通过使用大过量(10 equiv.)的 烯醇硅醚底物 39 以 $80 \%$ 的高收率成功得到交叉偶联产 物 40 (Scheme 12). 显然, 该反应的最大限制在于需要 使用过量的一分子烯醇硅醚底物. 为了解决上述问题, 有机化学家通过发展出利用双烯醇硅醚的方法将上述 分子间的反应变为分子内的反应, 并实现了良好的化学 选择性和立体选择性. 1998 年, Schmittel 等 ${ }^{[34]}$ 报道了利 用双烯醇硅醚实现了非对映选择性碳碳键的构建, 以 59\%的产率和 9:1 的非对映选择性顺利得到偶联产物 42 (Scheme 13). 虽然在该报道中仅仅展示了四个底物,

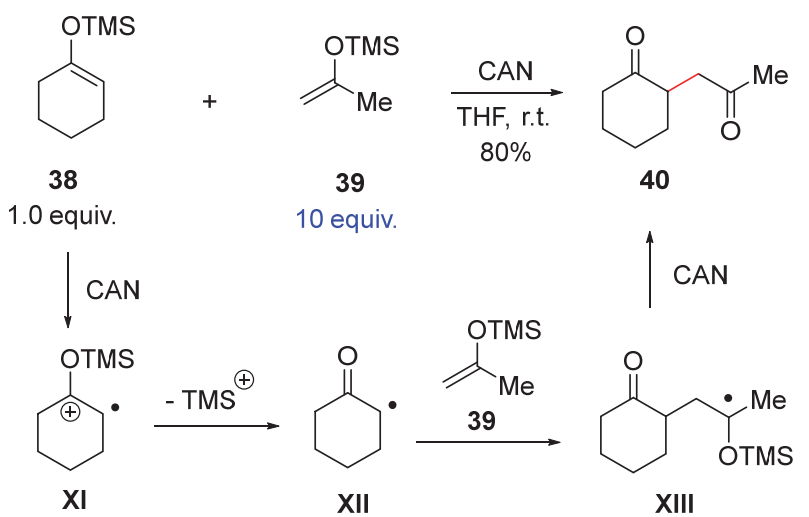

图式 12 烯醇硅醚分子间交叉氧化偶联反应

Scheme 12 Intermolecular oxidative cross-coupling reaction of enol silanes reported by Ruzziconi's group

但却为之后相关研究奠定了坚实的基础. 此外, 他们后 续还将反应底物拓展至钛、锆的烯醇硅醚，并对该类型 反应的机理和立体选择性进行了深入的研究 ${ }^{[35]}$.<smiles>C/C=C(\O[Si](C)(C)O/C(=C/C)c1ccccc1)c1ccccc1</smiles><smiles>CNC(C)NC(=O)O</smiles><smiles>CC(C(=O)c1ccccc1)C(C)C(=O)c1ccccc1</smiles>

41

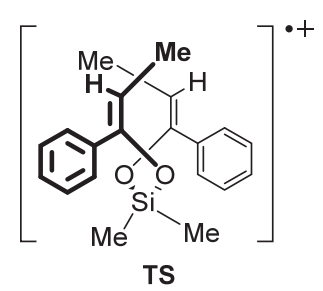

42
图式 13 首例双烯醇硅醚分子内氧化偶联反应

Scheme 13 First oxidative homo-coupling reaction of bis-enol silanes reported by Schmittel's group

基于 Schmittel 的研究工作, Thomson 研究团队 ${ }^{[36-38]}$ 在这一领域做了大量卓有成效的研究工作(Scheme 14), 并将双烯醇硅醚分子内氧化偶联反应成功应用于多个 天然产物的全合成中(详见第二节).

2007 年, Thomson 课题组 ${ }^{[36]}$ ]报道了双烯醇硅醚底物 43 在与 Schmittel 报道类似的反应条件下，可以实现分 子内的交叉氧化偶联反应, 并顺利构建产物 44 中的全 碳季碳中心. 2008 年, 他们 ${ }^{[37]}$ 还实现了环状双烯醇硅醚 底物 45 非对映选择性的交叉氧化偶联反应. 2009 年, 该 研究小组 ${ }^{[38]}$ 更是将该反应与经典的 1,4-Micheal 加成反 应串联, 成功实现了三组分的反应, 所得氧化偶联产物 49 在 Paal-Knorr 反应条件下可以顺利转化为吡咯类产 物 50. 更重要的是, 他们还将该反应作为关键合成策略 完成了天然产物 metacycloprodigiosin 和 prodigiosin R1 的不对称全合成. 


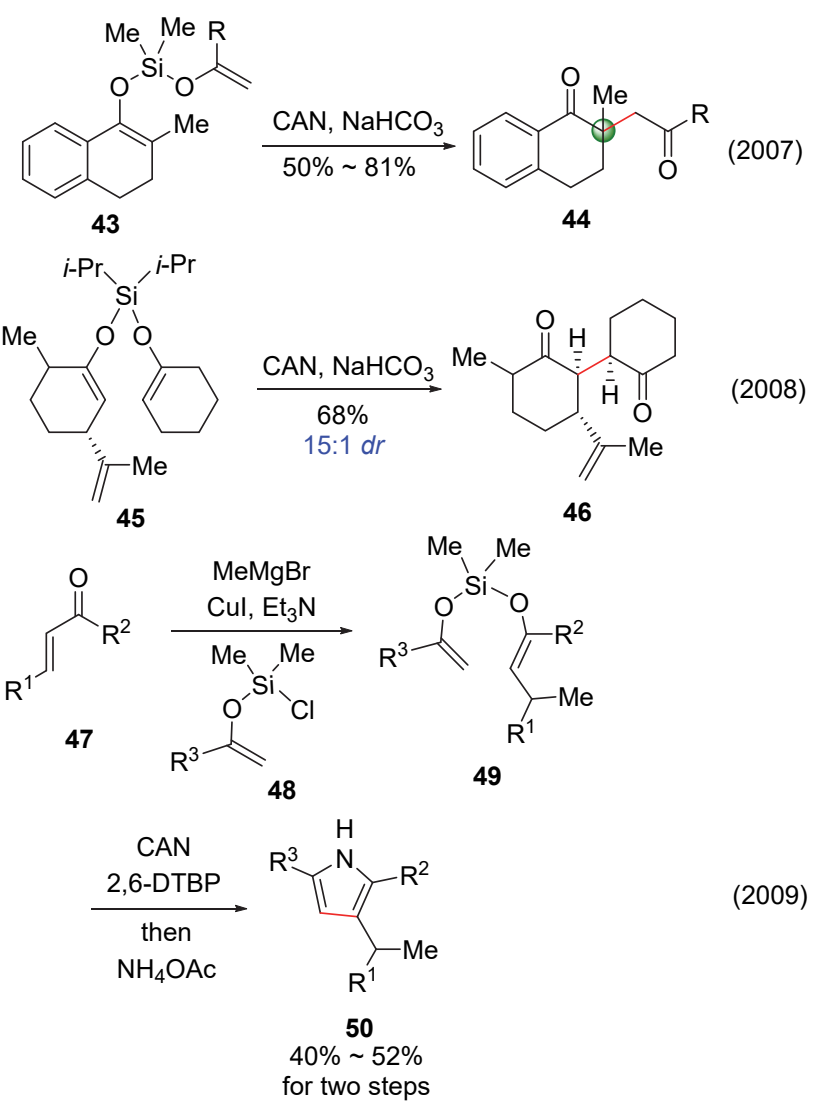

图式 14 Thomson 对双烯醇硅醚分子内氧化偶联反应的研究 Scheme 14 Intramolecular oxidative coupling reaction of bisenol silanes reported by Thomson's group

在 Schmittel、Thomson 等 ${ }^{[39]}$ 研究工作的启发下, 2019 年, 杨震和龚建贤课题组报道了利用 CAN 诱导的 双烯醇硅醚底物分子内交叉氧化偶联反应来非对映选 择性地构建连续全碳季碳手性中心和叔碳手性中心, 为 实现复杂天然产物左右两个不同片段的连接提供了一 种行之有效的合成策略(Scheme 15).

此外, 利用近些年最新发展的有机电化学合成方法 来实现烯醇硅醚的氧化偶联反应亦有相关报道.

2019 年, Hilt 等 ${ }^{[40]}$ 报道了不同环系的 $\beta$-羰基酯 $\mathbf{5 4}$ 与过量 (5.0 equiv.) 的烯醇硅醚底物 $\mathbf{5 5}$ 在催化量 (10 mol\%)锰盐作用下实现电化学合成碳碳键的方法, 并成 功构建偶联产物 56 中的全碳季碳中心. 不过, 目前该反 应的底物仍有一定的局限性, 同时通过添加手性配体也 未能实现相应产物对映选择性的合成(Scheme 16). 机 理方面, 反应体系中的低价态锰盐首先在阳极进行氧化 反应生成高价态的锰(IV)物种, 随后其将烯醇硅醚底物 55 氧化成自由基中间体 XIV; 另外, $\beta$-羊炭基酯 54 在阴极 所产生碱的作用下形成烯醇负离子中间体 XVI, 然后与 XIV 进行偶联反应得到另一自由基中间体 XVII，之后 其被锰(III)物种氧化并脱去硅基正离子生成最终产物

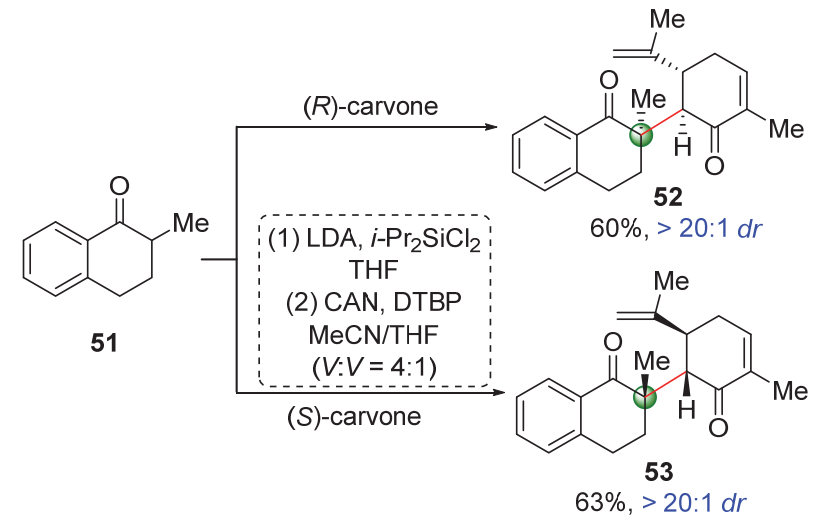

图式 15 立体选择性的双烯醇硅醚交叉氧化偶联反应

Scheme 15 Stereoselective oxidative cross-coupling reaction of bis-enol silanes reported by Yang and Gong's group

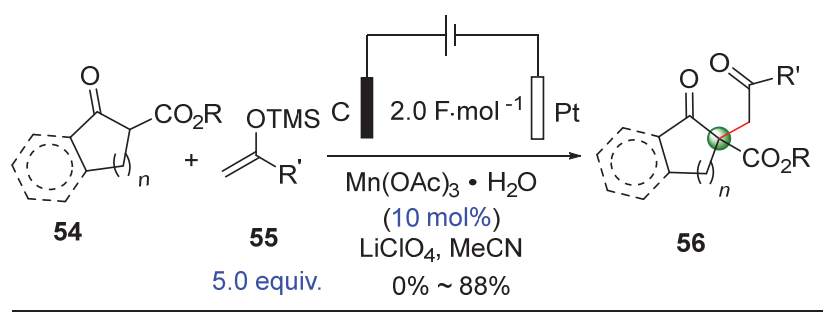<smiles>CCOC(=O)CC(CC(C)=O)(CC(C)(C)C)C(=O)CC1(C(=O)OCC)CCCCC1=O</smiles>

图式 16 锰(III)催化下烯醇硅醚的电化学合成反应

Scheme 16 Electrochemical, manganese-assisted $\mathrm{C}-\mathrm{C}$ bond formation reported by Hilt's group

56. 此外，反应过程中产生的烯醇硅醚自由基中间体 XIV 也可能进行自身二聚反应而得到副产物 57 (Scheme 17).

\section{3 烯胺的氧化偶联反应}

烯胺不仅是烯醇类化合物氧化偶联反应常见的底 物，同时还是实现有机小分子不对称催化形式反应的理 想底物. 早在 1992 年, Narasaka 等 ${ }^{[41]}$ 就报道了烯胺化合 物 58(1.0 equiv.) 和烯醇硅醚 $\mathbf{5 9}$ (2.0 equiv.) 在以 CAN 作 单电子氧化剂的条件下实现的交叉氧化偶联反应 (Scheme 18). 可见，在与前人使用的类似反应条件 下 ${ }^{[33]}$ ，烯胺会被优先氧化成自由基中间体 XVIII，从而 避免使用大过量的烯醇硅醚就可以实现相应交叉氧化 偶联反应. 不过，直到 2007 年，MacMillan 等 ${ }^{[42]}$ 才利用 有机小分子催化剂 64 实现了该反应的不对称催化形式, 并提出了全新的 “单占分子轨道催化(SOMO catalysis)” 有机催化理论，为实现烯醇类化合物对映选择性的交叉 氧化偶联反应提供了一种重要的合成方法(Scheme 19). 


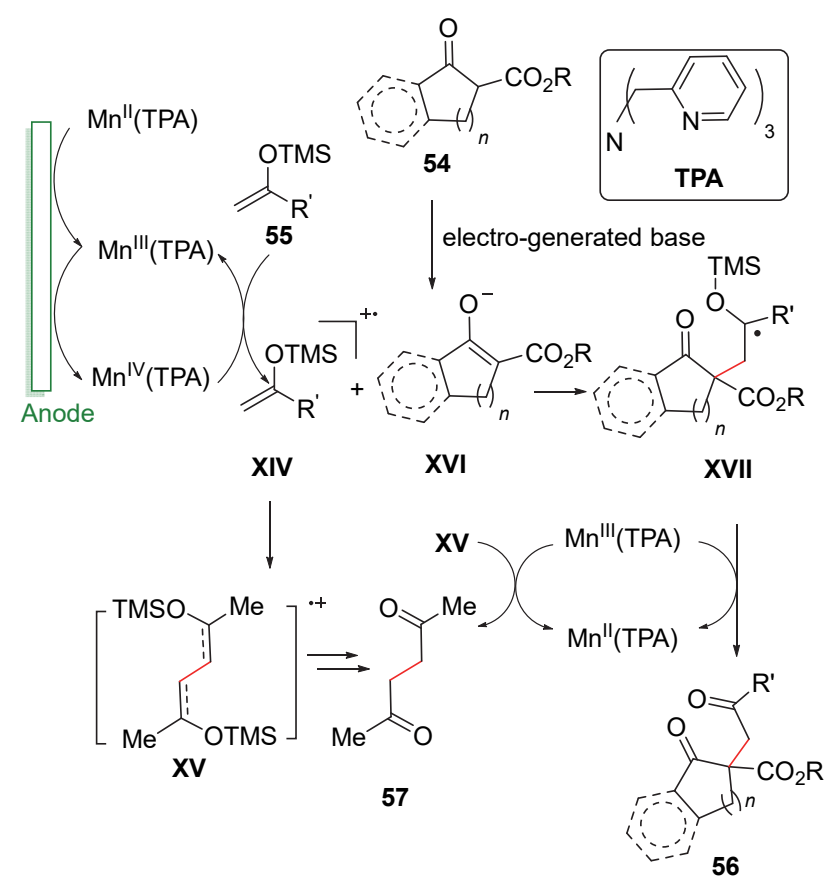

图式 17 电化学条件下烯醇硅醚分子间氧化偶联反应可能的 机理

Scheme 17 Proposed reaction mechanism for the Mn(TPA) dichloride mediated reaction reported by Hilt's group<smiles></smiles>

58 1.0 equiv.

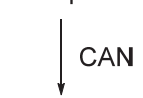<smiles>C=C(OCCCCCCCCC(C)(C)C)c1ccccc1</smiles>

XVIII<smiles>C=C([OH2+])c1ccccc1</smiles>

59 2.0 equiv.

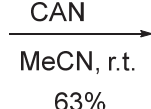
$63 \%$<smiles>CC(C)(C)C(=O)CCC(=O)c1ccccc1</smiles>

60<smiles>CCO</smiles>

图式 18 烯胺和烯醇硅醚分子间交叉氧化偶联反应

Scheme 18 Intermolecular oxidative cross-coupling reaction of enamine and enol silane reported by Narasaka's group

可见光促进的反应通常作为一种基于单电子转移 (SET)或能量转移(ET)的反应过程, 具有绿色、清洁、可 再生等诸多优点, 近些年来受到越来越多化学家的关 注，已发展成为当前最热门的研究领域之一 ${ }^{[43]}$.

2012 年, Akita 和 Koike 等 ${ }^{[44]}$ 报道了利用催化量(5 $\mathrm{mol} \%)$ 的光敏剂 $\left[\mathrm{Ru}(\mathrm{bpy})^{3}\right]^{2+}$ 在可见光的条件下实现了 烯胺 65 和烯醇硅醚 66 的交叉氧化偶联反应. 在该研究 中, 他们使用杜醌 68 作为催化循环中的电子受体, 并发 现路易斯酸 $\mathrm{LiBF}_{4}$ 可以促进电子的转移过程(Scheme
20).

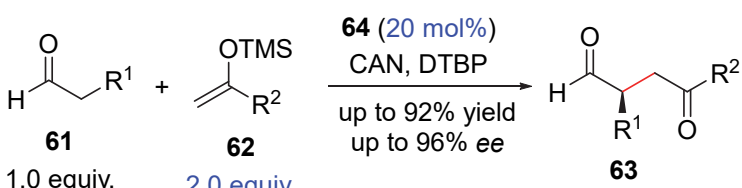

1.0 equiv. $\quad 2.0$ equiv.<smiles>CN1C(=O)C(Cc2ccccc2)NC1C(C)(C)C</smiles>

图式 19 有机催化下对映选择性的交叉氧化偶联反应 Scheme 19 Enantioselective organocatalytic oxidative crosscoupling reaction reported by MacMillan's group<smiles>[R]C=C([R9])N1CCOCC1</smiles>

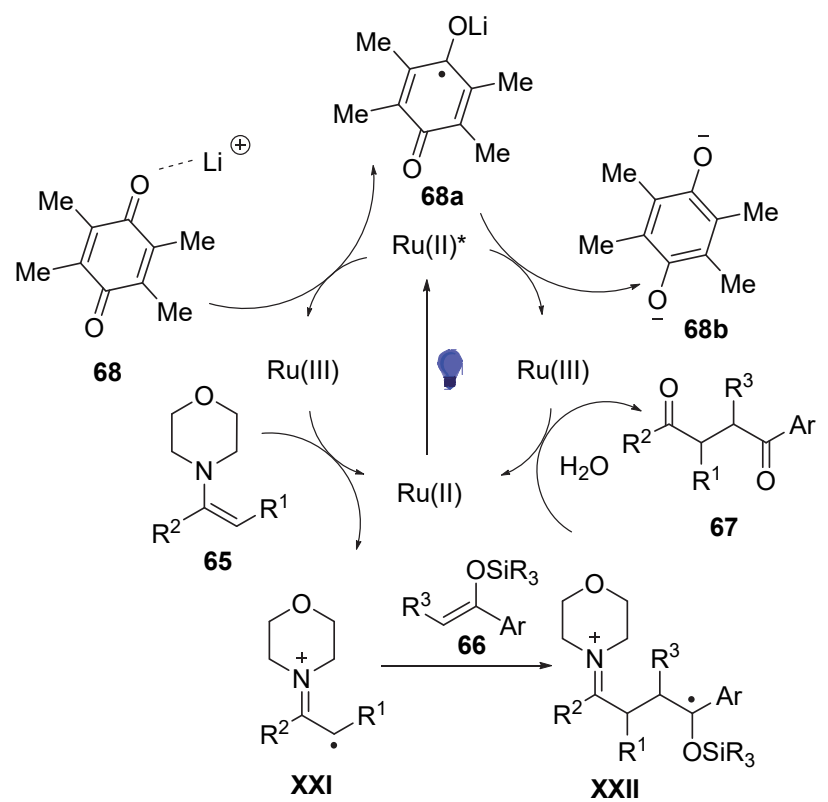

图式 20 可见光催化下烯胺的交叉氧化偶联反应 Scheme 20 Visible-light-promoted oxidative cross-coupling reaction of enamines reported by Akita and Koike's group

此外, 2010 年, 贾彦兴课题组 ${ }^{[45]}$ 报道了利用烯胺进 行自身氧化偶联反应得到对称形式吡咯 71, 并以此为 关键反应实现了天然产物 purpurone、 lamellarins D, H, R 和 ningalin $\mathrm{B}$ 的全合成. 在该反应过程中, 胺类底物 69 作为 Paal-Knorr 反应的合成前体, 首先与醛 70 反应生成 烯胺中间体 XXIII, 之后在单电子氧化剂的作用下进行 自由基自身二聚反应(Scheme 21). 2019 年，雷爱文课题 组 ${ }^{[46]}$ 更是报道利用最新发展的有机电化学合成方法成 功实现了与上述类似的反应过程. 


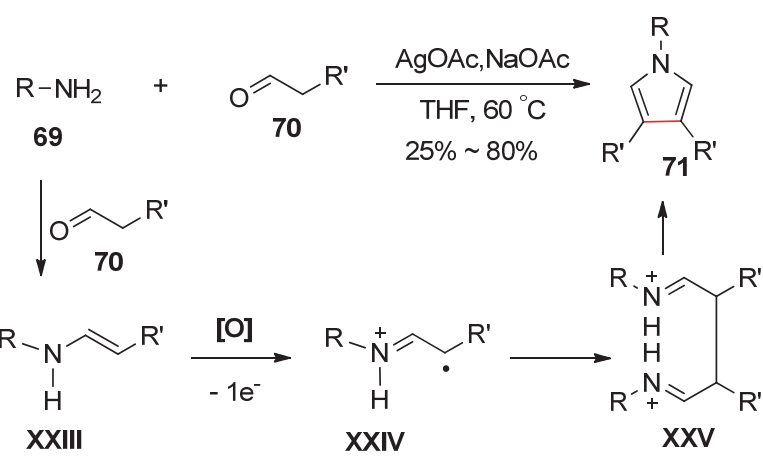

图式 21 利用烯胺自身氧化偶联反应一步合成吡咯 Scheme 21 Pyrroles synthesis via oxidative homo-coupling reaction of enamines reported by Jia's group

在 MacMillan 报道 ${ }^{[42]}$ 的烯胺对映选择性交叉氧化偶 联反应中 (Scheme 19), 仍需要使用化学计量比 (2.0 equiv.)的 CAN 作为反应的单电子氧化剂. 2018 年, Jørgensen 报道了使用催化量的氧化剂来实现有机小分 子催化下的 $\alpha, \beta$-不饱和醛 $\gamma$ 位的交叉氧化偶联反应. 他 们使用 74 作为有机小分子催化剂, 催化量 $(20 \mathrm{~mol} \%$ )的 铜盐作单电子氧化剂, 空气作终氧化剂, 成功实现了偶 联产物 75 中碳碳键立体选择性的构建. 不过, 为实现该 交叉氧化偶联反应，仍需使用稍过量(3.0 equiv.)的一分 子 $\alpha, \beta$-不饱和醛 73 (Scheme 22) $)^{[47]}$.<smiles>[X]c1ccccc1/C(=C/C=O)CCC</smiles>

72 1.0 equiv.<smiles>[R]c1ccc2c(c1)/C(=C/C=O)CCC2</smiles>

73 3.0 equiv.

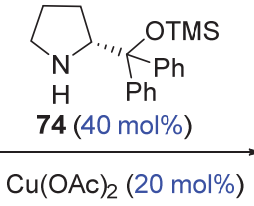

$\mathrm{DCM}$, air, r.t. up to $76 \%$ yield up to $>99 \%$ ee<smiles>[R1]c1ccccc1/C(=C/C=O)C1CCc2ccccc2C1=CC=O</smiles>

75

图式 22 不对称催化下 $\alpha, \beta$-不饱和醛 $\gamma$ 位交叉氧化偶联反应 Scheme 22 Catalytic asymmetric oxidative $\gamma$-coupling of $\alpha, \beta$ unsaturated aldehydes reported by Jørgensen's group

\section{4 其他烯醇类化合物的氧化偶联反应}

除了前面提到的经典烯醇形式底物、(双)烯醇硅醚、 烯胺外, 硼烯醇化合物的氧化偶联反应亦有相关研究报 道. 2014 年, Hirao 研究小组 ${ }^{[48]}$ 利用化学计量比的钒盐作 单电子氧化剂, 成功实现了嗍烯醇化合物自身氧化偶联 反应, 不过该反应的非对映选择性相对较低 $(\mathrm{dl}:$ meso $=67: 33)$. 其中, 反应中间体 77 由烯酮底物 76 通过
1,4-硼氢化反应得到(Scheme 23).

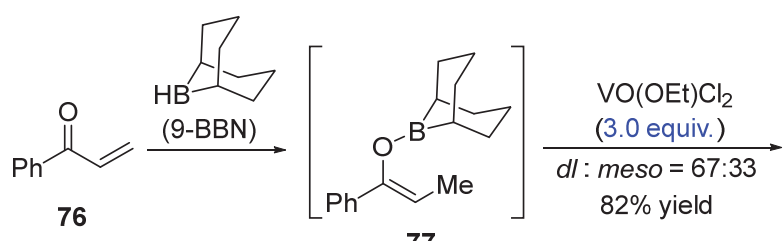<smiles>CC(C(=O)c1ccccc1)C(C)C(=O)c1ccccc1</smiles>

图式 23 嗍烯醇化合物自身氧化偶联反应

Scheme 23 Oxidative homo-coupling of boron enolate reported by Hirao's group

2015 年, 他们报道了利用化学计量比 1：1 的硼烯 醇化合物 79 与烯醇硅醚 80 在之前报道的条件下可以实 现化学选择性的交叉氧化偶联反应, 并且能顺利构建产 物 81 中的全碳季碳中心. 究其原因, 主要是因为两种烯 醇类化合物的氧化还原电势不一样，单电子氧化剂钒盐 会优先氧化嗍烯醇化合物 79, 而烯醇硅醚 80 则作为自 由基受体(Scheme 24) ${ }^{[49]} .2017$ 年, 该研究小组 ${ }^{[50]}$ 还进一 步把该反应的底物范围扩展至酯、酰胺等, 充分体现了 嗍烯醇化合物氧化偶联反应广泛的底物适用性.

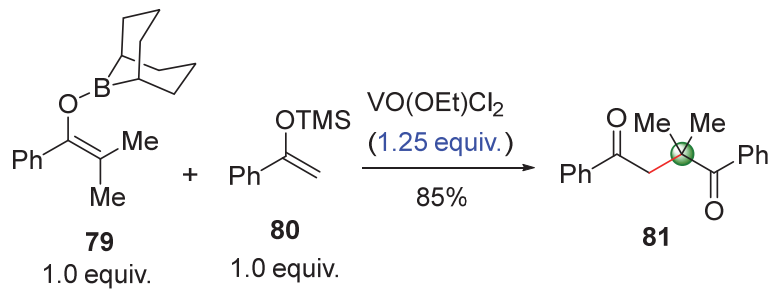

图式 24 硼烯醇化合物交叉氧化偶联反应

Scheme 24 Oxidative cross-coupling of boron enolate reported by Hirao's group

2020 年, 他们 ${ }^{[51]}$ 更是利用催化量的钒盐作单电子 氧化剂来实现上述化学转化, 并采用电子自旋共振谱法 (ESR) 和 ${ }^{51} \mathrm{~V} \mathrm{NNR}$ 技术研究了钒的变化(Scheme 25).

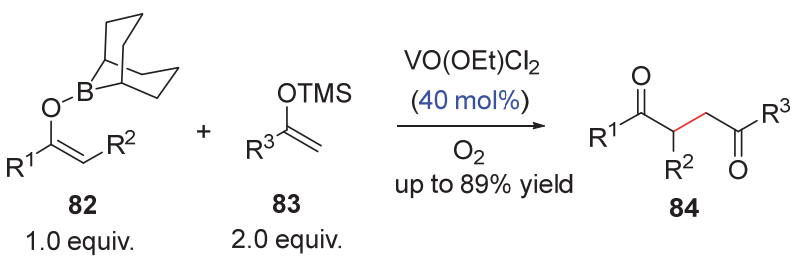

图式 25 钒(V)催化下嗍烯醇化合物交叉氧化偶联反应 Scheme 25 Oxovanadium(V)-catalyzed oxidative cross-coupling of boron enolates reported by Hirao's group

\section{2 烯醇类化合物氧化偶联反应在天然产物全合 成中的应用}

烯醇类氧化偶联反应作为 $1,4-$ 二羰基骨架的高效合 
成方法, 同时无需预先对参与反应的碳原子位点进行官 能团化便能实现 $\mathrm{C}-\mathrm{C}$ 键的直接构建, 可显著提高天然 产物全合成的效率. 根据反应的类型, 按照分子间的氧 化偶联反应和分子内的氧化偶联反应分别进行简要概 述.

\section{1 分子间的氧化偶联反应}

早在 1988 年, Belletire 等[52]报道了利用烯醇类化合 物分子间氧化偶联反应作为关键合成策略来实现木脂 素类天然产物 $( \pm)$-wikstromol 的消旋全合成. 首先, 羧 酸 85 在 LDA 作碱、单质碘作氧化剂的条件下进行自身 氧化偶联反应, 之后在乙酸酐加热回流条件下得到产物 86, 所得酸酐 86 经醇解、选择性还原、酸化等步骤生成 高级中间体 87 , 最后经数步官能团转化完成 $( \pm)$-wikstromol 的全合成(Scheme 26).

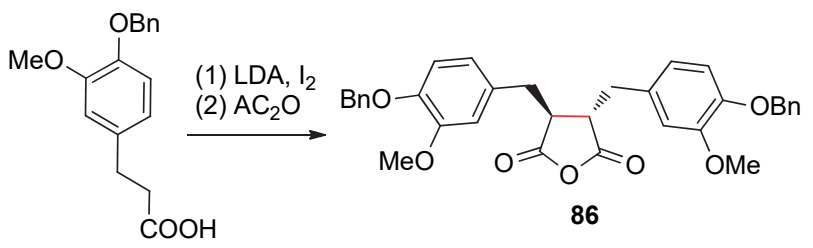

85

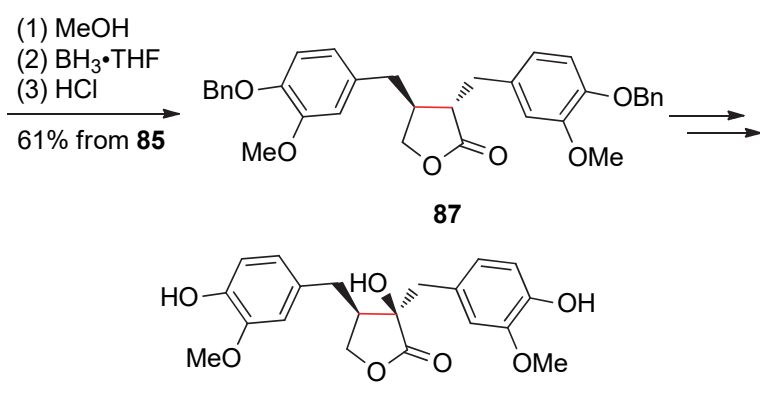

$( \pm)$-wikstromol

图式 26 天然产物 $( \pm)$-wikstromol 的全合成

Scheme 26 Total synthesis of $( \pm)$-wikstromol reported by Belletire's group

2006 年, Baran 等 ${ }^{[11]}$ 利用自己课题组发展的烯醇类 化合物交叉氧化偶联反应成功实现木脂素类天然产物 (一)-bursehernin 的不对称全合成. 在标准的反应条件 下, 噁唑烷酮 88 与酯 89 可以顺利反应生产偶联产物, 随后用硼氢化锂化学选择性地脱除手性辅基并在 1,5-二 氮杂二环 $[5.4 .0]+$ 一烯-5 (DBU)作碱加热的条件下实现 内酯化反应, 最后以 $41 \%$ 的总收率完成 $(-)$-bursehernin 的对映选择性全合成(Scheme 27) ${ }^{[19]}$. 此外, 该小组还采 用类似的合成策略，先后完成了一种基质金属蛋白酶 (MMPs)抑制剂和潜在抗肿瘤小分子药物 BMS-906024 的高效合成 ${ }^{[11 \mathrm{c}, 53]}$.

2008 年, Baran 等 ${ }^{[54]}$ 进一步发展出吲哚类底物 90 与 光学纯香芹酮底物 91 立体选择性的分子间交叉氧化偶<smiles>O=C(CCc1ccc2c(c1)OCO2)N1C(=O)OCC1P</smiles><smiles>COc1ccc(CCC(=O)OC(C)(C)C)cc1OC</smiles>

$$
\begin{aligned}
& \text { (1) } \mathrm{LDA}^{\mathrm{Cu}} \mathrm{Cu}(\mathrm{II}) \\
& \text { (2) } \mathrm{LiBH}_{4} \\
& \text { (3) } \mathrm{DBU}^{-} \Delta \\
& \begin{array}{l}
41 \% \text { for } 3 \text { steps } \\
\text { single isomer }
\end{array}
\end{aligned}
$$

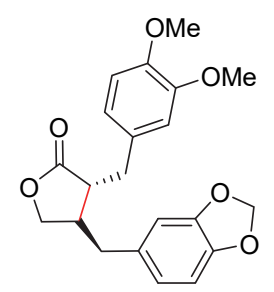

(-)-bursehernin
图式 27 天然产物(一)-bursehernin 的全合成

Scheme 27 Total synthesis of (-)-bursehernin reported by Baran's group

联反应. 更重要的是, 利用该反应作为关键合成策略, 他们顺利完成了 hapalindoles、fischerindoles、welwitindoles 家族多个生物碱简洁高效的不对称全合成 (Scheme 28), 为后续相关生物活性研究奠定了坚实的 基础 ${ }^{[55]}$.<smiles>c1ccc2[nH]ccc2c1</smiles>

90<smiles>C=C(C)[C@]1([Hg])CC=C(C)C(=O)[C@H]1Br</smiles>
91
LiHMDS Cu"-2ethylhexanoate. THF, 53\%<smiles>C=CC1CCC(C(C)C)C(c2c[nH]c3ccccc23)C1[N+](=O)[O-]</smiles>

hapalindole D<smiles>C=C[C@H]1CC[C@H]2[C@@H](C)[C@H](C)c3[nH]c4ccccc4c3[C@H]2[C@H]1C#N</smiles>

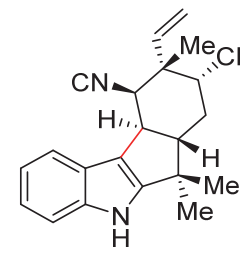

fischerindole $\mathrm{G}$

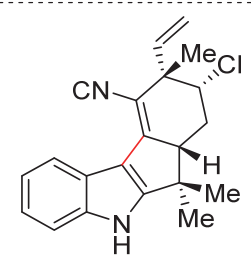

fischerindole I

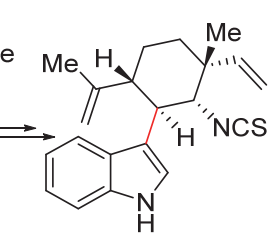

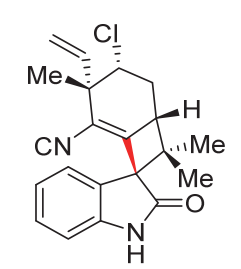

welwitindolinone $A$

图式 28 hapalindoles、fischerindoles、welwitindoles 家族天然 产物的不对称全合成

Scheme 28 Enantiospecific total synthesis of hapalindoles, fischerindoles, welwitindoles reported by Baran's group 
2009 年, Baran 等[56]报道了烯醇 93 和烯醇硅醚 94 在 CAN 作单电子氧化剂的条件下进行分子间交叉偶联 反应得到产物 95 , 然后经数步化学转化生成高级中间 体 96, 最后利用分子内 Diels-Alder 反应顺利实现天然 产物 maoecrystal V 核心骨架的构建(Scheme 29).<smiles>CC1(C)C=CC(=O)C(=CO)C1</smiles>

93

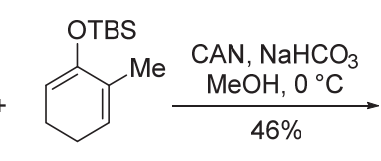

94

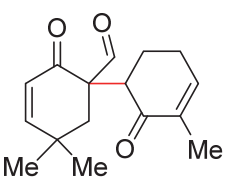

95<smiles>C=CC(=O)OCC1C=CCC(C)(OC(C)=O)C1C1CC(C)(C)C=CC1=O</smiles>

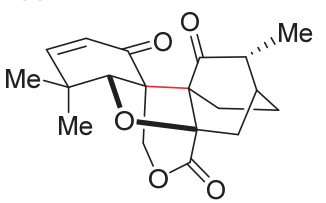

maoecrystal $\mathrm{V}$

图式 29 天然产物 maoecrytal V 核心骨架的构建

Scheme 29 Synthesis of the core carbon skeleton of maoecrystal V reported by Baran's group

2011 年, Thomson 等 ${ }^{[22]}$ 利用自己课题组发展的轴手 性联苯酚高效合成方法学成功实现了咔唑类天然产物 bis- murrayaquinone A 的不对称全合成. 在 $\mathrm{LDA} / \mathrm{CuCl}_{2}$ 的反应条件下, 光学纯底物 15 经分子间氧化偶联反应 可以得到单一的偶联产物 16, 随后进行芳构化生成手 性联苯酚 17 , 之后经过 3 步简单的化学转化得到高级中 间体 98, 最后在钯(II)催化的条件下构建分子中的咔唑 骨架结构以及使用 $\mathrm{CAN}$ 氧化脱除甲基, 最终完成 bismur- rayaquinone A 的全合成(Scheme 30).

从海洋细菌中分离得到的 lomaiviticins 家族天然产 物(如 lomaiviticin A、 lomaiviticin B)因其独特的结构特 征 $\left(C_{2}\right.$-对称)和优异的生物活性(抗菌、抗肿瘤活性) ${ }^{[57]}$, 吸引了全球有机化学家的广泛关注. 结构上, lomaiviticin A 和 lomaiviticin B 的左右两个片段通过中间立体 拥挤的 $\mathrm{C}-\mathrm{C}$ 键连接(Scheme 31). 因此，如何实现该 $\sigma$ 碳碳键立体选择性的构建是实现 lomaiviticins 家族天然 产物全合成的关键所在.

2008 年, Shair 等 ${ }^{[58]}$ 报道了手性酮底物 99 的烯醇锂 盐在铁盐作单电子氧化剂的条件下, 以中等收率得到单 一的自身氧化偶联产物 100 , 之后经数步简单的官能团 转化便可以顺利构建 lomaiviticins 家族天然产物的核 心骨架(Scheme 32). 2010 年, 他们 ${ }^{[59]}$ 还利用同样的方法
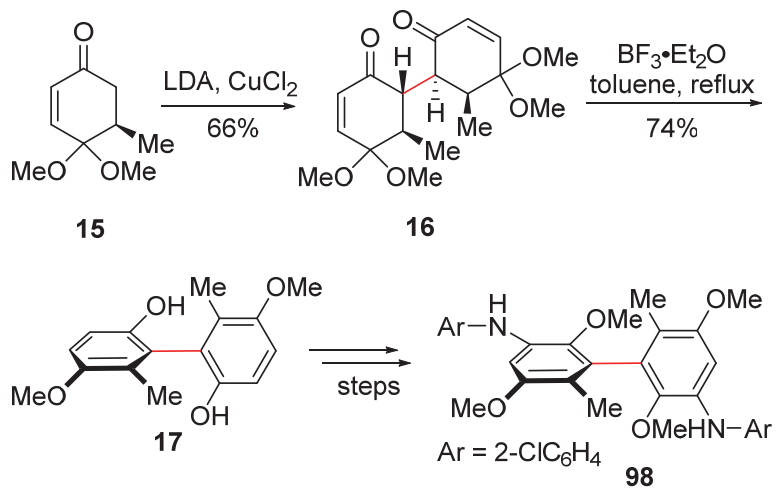

(1) $\mathrm{Pd}(\mathrm{OAc})_{2}$, $\left[\mathrm{HPt}-\mathrm{Bu}_{3}\right]\left[\mathrm{BF}_{4}\right]$ $\mathrm{NaOt}-\mathrm{Bu}, 160^{\circ} \mathrm{C}$ microwave, $73 \%$

(2) CAN, $95 \%$

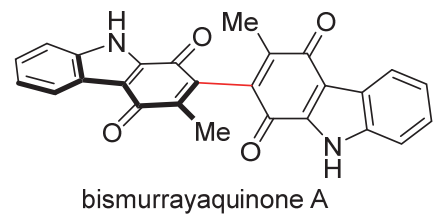

图式 30 天然产物 bismurrayaquinone A 的全合成 Scheme 30 Total synthesis of bismurrayaquinone A reported by Thomson's group

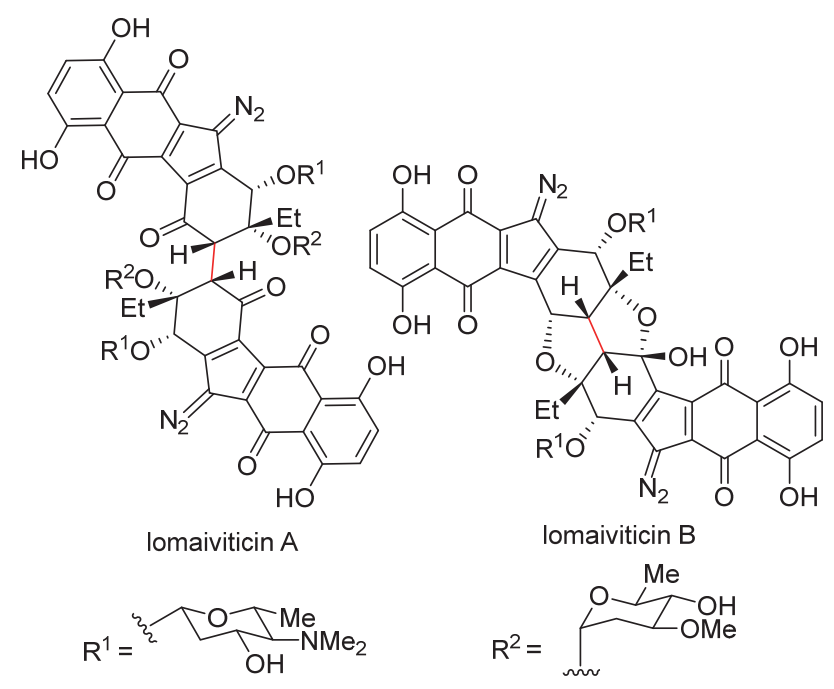

图式 31 天然产物 lomaiviticin A、 lomaiviticinB 的结构 Scheme 31 Structures of lomaiviticin A and lomaiviticin B 实现 lomaiviticins 家族分子中多环体系骨架的构建.

2011 年, Herzon 等[60]报道利用烯醇硅醚自身氧化偶 联反应实现了更为复杂环系底物的二聚反应，并成功构 建 lomaiviticins 家族天然产物分子中立体拥挤的 $\mathrm{C}-\mathrm{C}$ 键，最后使用三氟乙酸脱除保护基即可得到天然产物糖 苷配基(Scheme 33).

2015 年, Bisai 等[61]报道了一种无需过渡金属参与 下 2-氧化吲哚底物 106 自身氧化偶联反应, 并顺利构建 产物 107 中的连续全碳季碳中心. 虽然该反应的非对映 选择性较低(1.2：1 dr), 但为实现生物碱 $( \pm)$-folicanthine 的消旋全合成提供了一种高效的合成手段(Scheme 34). 


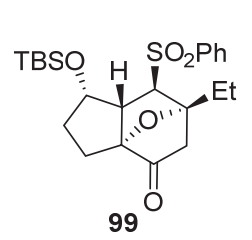

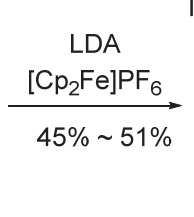

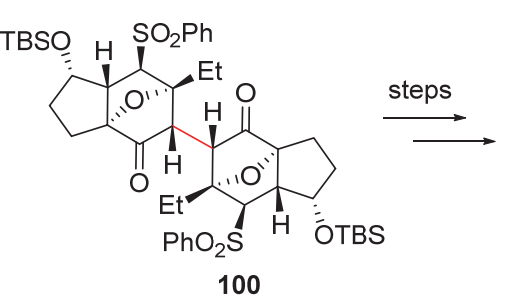

100

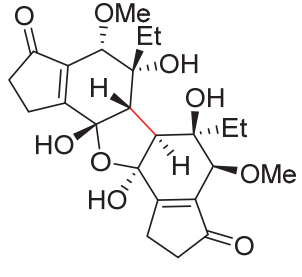

101

图式 32 lomaiviticins 家族天然产物核心骨架的合成

Scheme 32 Synthesis of the core ring system of lomaiviticins reported by Shair's group

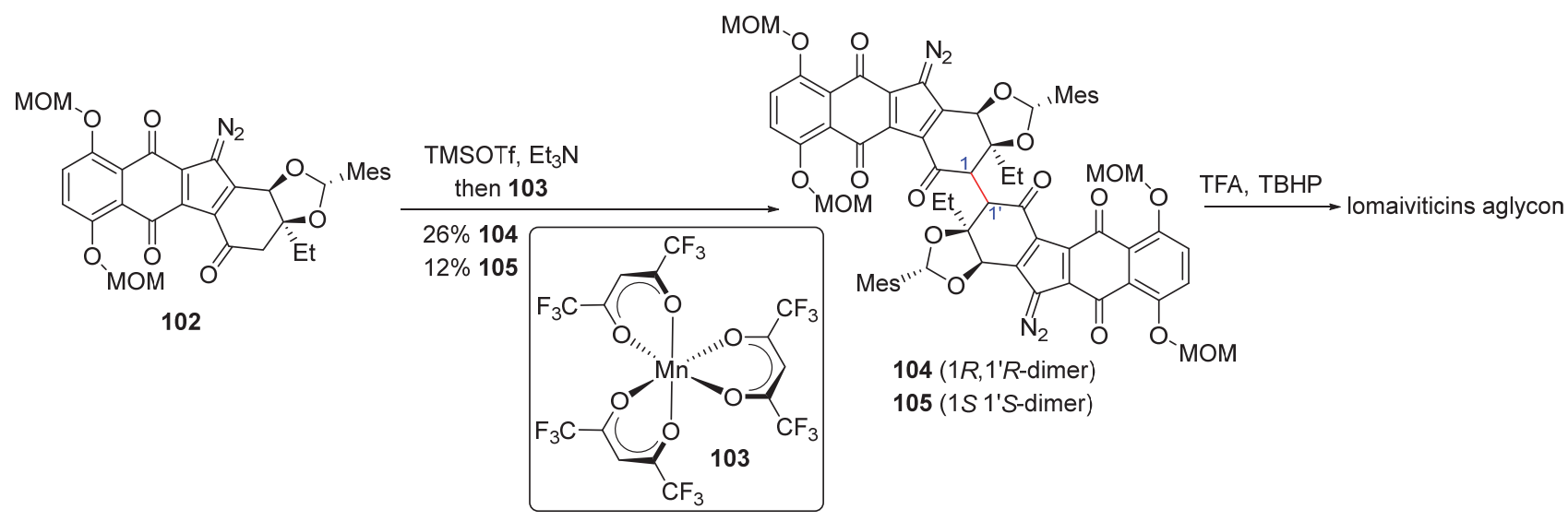

图式 33 lomaiviticins 家族天然产物糖苷配基的合成

Scheme 33 Enantioselective synthesis of lomaiviticins aglycon reported by Herzon's group

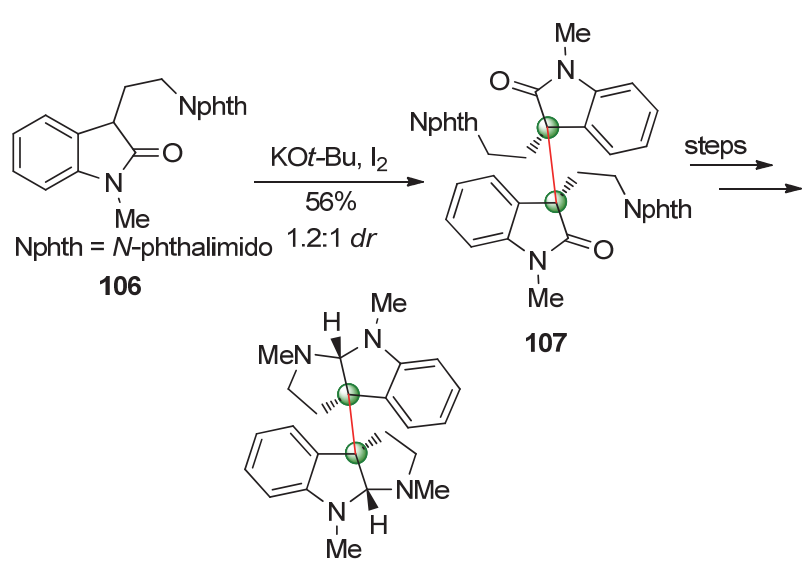

(土)-folicanthine

图式 34 天然产物 $( \pm)$-folicanthine 的全合成

Scheme 34 Total synthesis of $( \pm)$-folicanthine reported by Bisai's group

2015 年, 杨震和陈家华课题组 ${ }^{[62]}$ 报道了利用烯醇 硅醚分子间交叉氧化偶联反应作为关键合成策略实现 了五味子家族三萜类天然产物 propindilactone $\mathrm{G}$ 的不对 称全合成. 光学纯的高级中间体 108 在 CAN 作单电子 氧化剂的条件下与另一分子烯醇硅醚 109 反应顺利得到 交叉偶联产物 110, 之后利用 Horner-WadsworthEmmons 反应延长分子中的侧链得到目标产物 111, 最
后在 $\mathrm{OsO}_{4} / \mathrm{NMO}$ 条件下发生双羟化/内酯化串联反应完 成天然产物 propindilactone $\mathrm{G}$ 的全合成(Scheme 35).

\section{2 分子内的氧化偶联反应}

早在 1992 年, Cohen 和 Ramig 等[63]在( \pm )-hirsutene 的全合成工作中就将烯醇碱金属盐分子内交叉氧化偶 联反应作为关键的合成策略. 左右两个不同片段 112 和 114 通过共轭加成可以得到双烯醇锂盐 115, 之后在铁 盐作单电子氧化剂的条件下进行分子内氧化偶联反应, 以 $64 \%$ 的高收率得到单一的偶联产物 116, 然后使用兰 尼镍(Ra-Ni)还原反应脱除硫取代基生成 117 , 最后经数 步官能团转化完成( \pm )-hirsutene 的全合成(Scheme 36).

2005 2006 年, Baran 等[11]先后报道了使用烯醇类 化合物分子内氧化偶联反应成功实现了吲哚类生物碱 stephacidin A、avrainvillamide 和 stephacidin B 的不对称 全合成. 光学纯哌嗪二酮底物 118 在他们课题组发展的 $\mathrm{LDA} / \mathrm{Fe}(\mathrm{acac})_{3}$ 经典氧化条件下，以 $41 \%$ 的收率顺利得 到偶联产物 119, 之后通过脱除氮上 MOM 保护基、甲 酯的转化、形式 ene 反应等过程实现 stephacidin A 的全 合成，而 avrainvillamide 可以从 stephacidin A 通过 2 步 转化即得; stephacidin B 则通过 avrainvillamide 自发的可 逆二聚反应得到(Scheme 37) ${ }^{[64]}$. 


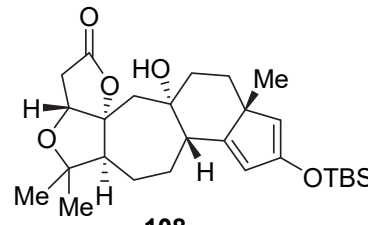

108

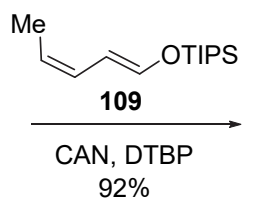

2.0:2.0:1.1:1.0 $d r$

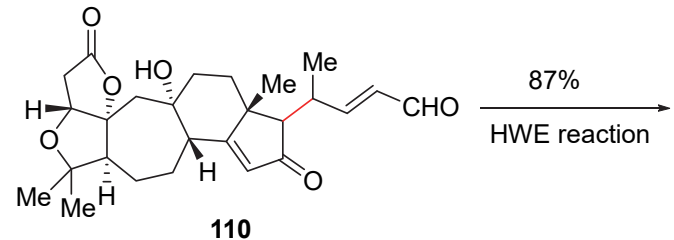<smiles>CCOC(C)=CC=CC(C)C1C(=O)C=C2C3CCC4C(C)(C)OC5(CCC21C)CC(=O)OC45CC3O</smiles><smiles>CO[N+](=O)[O-]</smiles>

图式 35 天然产物 propindilactone $\mathrm{G}$ 的全合成

Scheme 35 Total synthesis of propindilactone G reported by Yang and Chen's group<smiles>[X]C1=CCCC1=O</smiles>

112

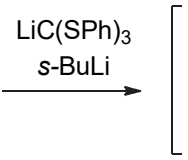<smiles>CC1=C(O)CCC1C([Mg])Cl</smiles>

113

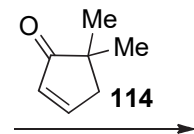

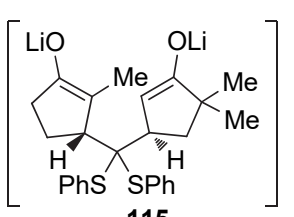

115

$\mathrm{FeCl}_{3} \downarrow 64 \%$

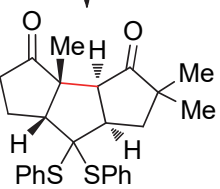

116

图式 36 天然产物 $( \pm)$-hirsutene 的全合成

Scheme 36 Total synthesis of $( \pm)$-hirsutene reported by Cohen and Ramig's group

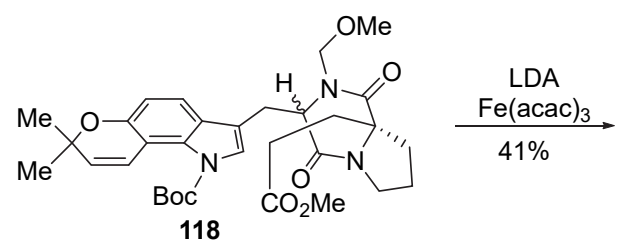

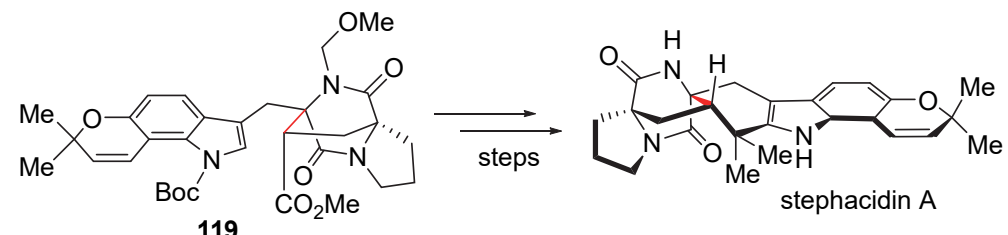
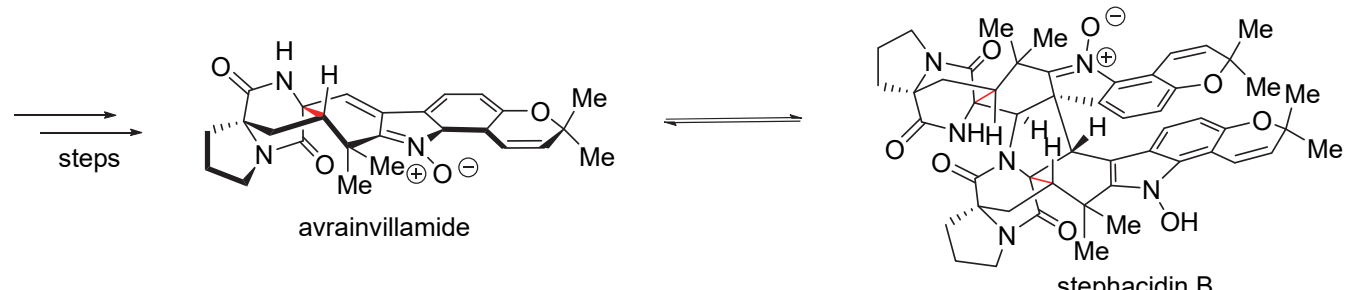

图式 37 天然产物 stephacidin A、avrainvillamide 和 stephacidin B 的全合成

Scheme 37 Total synthesis of stephacidin A, avrainvillamide and stephacidin B reported by Baran's group

上述 Baran 的研究工作展示了烯醇类化合物分子内 交叉氧化偶联反应在生物碱全合成方面的独特性和有 效性, 为后续相关天然产物的全合成研究提供了很好的 参考和借鉴. 比如, 2008 2010年, Overman 等 ${ }^{[65]}$ 在对吲 哚类生物碱 $( \pm)$-actinophyllic acid 的全合成研究工作中
就采取了类似的合成策略来构建天然产物分子中的 C$\mathrm{C}$ 键. 值得一提的是, 该关键反应还能在十克级别的规 模下进行，且产率无显著的降低，可见该反应十分高效， 具有广阔的应用前景(Scheme 38). 
<smiles>CC(C)(C)OC(=O)C(C(=O)OC(C)(C)C)c1[nH]c2ccccc2c1C1C(=O)CCC[N+]1(C)C(C)(C)C</smiles>
120

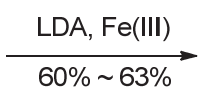
(up to $10 \mathrm{~g}$ scale)

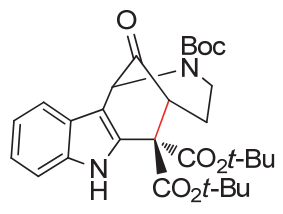

121

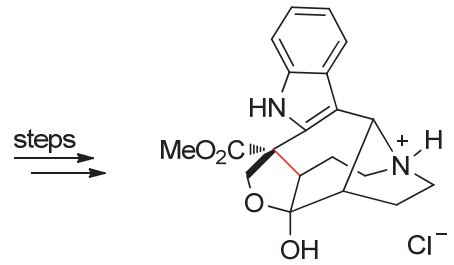

( \pm -actinophyllic acid

图式 38 天然产物 $( \pm)$-actinophyllic acid 的全合成

Scheme 38 Total synthesis of ( \pm )-actinophyllic acid reported by Overman's group<smiles>O=C(Cc1ccccc1[N+](=O)[O-])N(CCc1c[nH]c2ccccc12)[C@H](CO[Sb])c1ccccc1</smiles>

122

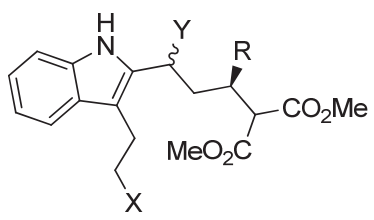

124

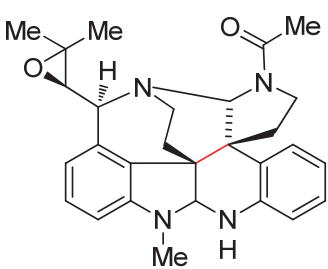

(-)-communesin A

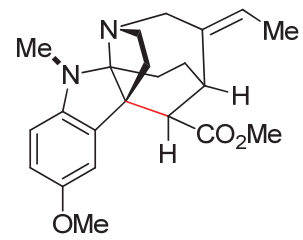

(-)-vincorine
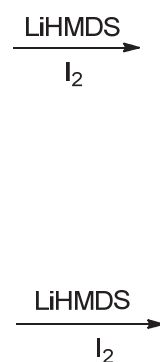<smiles>CCOC[C@H](c1ccccc1)C1CC[C@@]2(C=Nc3ccccc32)C(c2ccccc2[N+](=O)[O-])C1=O</smiles>

123

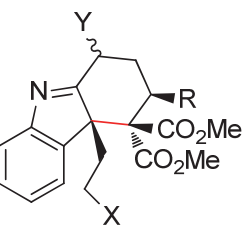

125 (type 1)

(type 2)

图式 39 两种不同类型的分子内氧化偶联反应在多个吲哚类生物碱全合成中的应用

Scheme 39 Oxidative coupling strategies for the synthesis of indole alkaloids reported by Ma's group

2010 2014 年, 马大为课题组 ${ }^{[66]}$ 对一系列具有重 要生理活性的吲哚类生物碱进行了全合成研究, 并取得 了卓有成效的研究成果. 他们发展出两种不同类型的分 子内氧化偶联反应来实现吲哚类生物碱核心骨架中 C$\mathrm{C}$ 键高效构建的合成方法, 并成功应用在天然产物 $(+)-$ communesins A, B, F、( $(-)$-vincorine、 $( \pm)$-aspidoylline A、 (+)-methyl $N$-decarbomethoxy-chanofruticosinate 等的全 合成研究工作中, 充分展示了该类型反应在吲哚类生物 碱天然产物全合成方面的独特优势(Scheme 39). 此外, 唐叶峰课题组对生物碱 spirobacillenes A 和 B 的全合成
研究 ${ }^{[67] 、 H i g u c h i ~}$ 对生物碱 scholarisine A 的全合成研 究 ${ }^{[68]}$ 等同样采用了上述类似的吲哚类底物分子内氧化 偶联反应来实现天然产物中 $\mathrm{C}-\mathrm{C}$ 键的高效构建.

前面提到利用双烯醇硅醚可以实现把分子间的反 应变成分子内的反应 ${ }^{[34-39]}$, 该合成策略在天然产物全合 成中具有重要的意义. 2018 年, Thomson 等报道了利用 双烯醇硅醚化合物分子内交叉氧化偶联反应来高效构 建分子中 $\mathrm{C}-\mathrm{C}$ 键, 并成功实现了海洋二萜类天然产物 $(+)-7,20$-diisocyanoadociane 的形式全合成. 他们利用 自己课题组发展的方法学 ${ }^{[36-38]}$, 首先将光学纯的酮转化 
为双烯醇硅醚化合物 $\mathbf{1 2 6}$, 在 $\mathrm{CAN} / \mathrm{NaHCO}_{3}$ 的条件下可 以实现 $\mathrm{C}-\mathrm{C}$ 键立体选择性的构建 $(d r$ 值为 $7: 1)$, 之后 在标准的关环烯烃复分解反应 $(\mathrm{RCM})$ 条件下得到三环 体系产物 127, 随后经过 $\mathrm{Pd} / \mathrm{C}$ 氢化还原、Wittig 反应、 Riley 氧化以及 Dess-Martin 氧化等常见的化学转化得到 高级中间体 128, 然后采用 Yoon 等 ${ }^{[69]}$ 在 2011 年报道的 可见光促进的还原偶联反应成功构建 129 中的四环体 系, 并可以得到单一的立体异构体, 最后经过已知的 3 步反应完成 $(+)$-7,20-diisocyanoadociane 的形式全合成 (Scheme 40 $)^{[70]}$. 此外, 该课题组此前还利用类似的双烯 醇硅醚化合物分子内交叉氧化偶联反应先后完成了复 杂天然产物 metacycloprodigiosin、prodigiosin R1、(土)propolisbenzofuran B 的全合成 ${ }^{[38,71]}$.<smiles>C=C(C)C1C=CC(O[Si](C(C)C)(C(C)C)C(C)C)=C[C@@H]1C(=C)C</smiles>

126

\section{(1) $\mathrm{CAN}, \mathrm{NaHCO}_{3}$} 7:1 $d r$

(2) $10 \mathrm{~mol} \%$ Grubbs II $41 \%$ ( 2 steps)

127

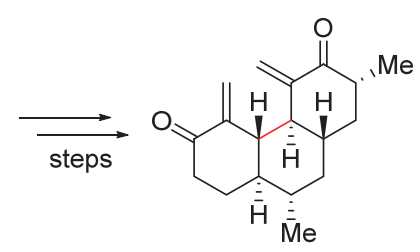

128
$3.5 \mathrm{~mol} \% \mathrm{Ru}(\mathrm{bpy})_{3} \mathrm{Cl}_{2}$ $\mathrm{HCO}_{2} \mathrm{H}, i-\mathrm{Pr}_{2} \mathrm{NEt}$ \begin{tabular}{c}
$\mathrm{h} v$ \\
\hline $92 \%$ \\
single isomer
\end{tabular}

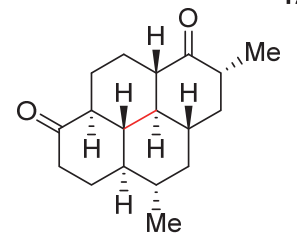

129

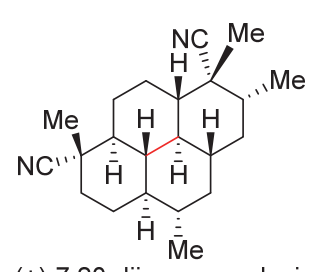

(+)-7,20-diisocyanoadociane
图式 40 天然产物(+)-7,20-diisocyanoadociane 的全合成 Scheme 40 Total synthesis of (+)-7,20-diisocyanoadociane reported by Thomson's group

\section{4 总结及展望}

烯醇类化合物氧化偶联反应作为 $\mathrm{C}-\mathrm{C}$ 键直接构建 和 1,4-二羰基骨架高效合成的手段, 近些年来受到有机 化学家的广泛关注, 并取得了快速的发展. 因此, 本文 在系统总结烯醇类化合物氧化偶联反应从 1935 年被首 次报道至今八十多年发展历史的基础上, 着重概述了从 2010 年至今近十年来的最新研究进展, 包括该反应在 有机合成方法学方面的发展及其在天然产物全合成方 面的应用, 以期为烯醇类化合物氧化偶联反应未来的进 一步发展提供参考和借鉴.

尽管目前烯醇类化合物氧化偶联反应的发展已经 取得了重要的突破, 但该反应依然存在不少亟待解决的
问题和挑战: (1)虽然目前烯醇类化合物分子间交叉氧化 偶联反应已有不少报道, 但能够实现物质的量比 $1: 1$ 下不同底物分子间的交叉氧化偶联反应例子并不多，而 且这些反应底物的限制性通常较大，产率也较低. (2)立 体专一性的烯醇类化合物氧化偶联反应依然是未来发 展的重点和难点之一, 特别是如何利用该反应来实现相 邻连续全碳季碳手性中心的高效构建. (3)目前很多烯醇 类化合物氧化偶联反应依然需要使用化学计量比的单 电子氧化剂, 而实现该类型反应催化形式下的高效合成 是未来 “绿色化学” 的必然要求. (4)最新发展的化学合 成方法(如有机光化学合成、有机电化学合成等)使用在 烯醇类化合物氧化偶联反应的报道并不多，尤其是其在 天然产物全合成中的应用更少.

\section{References}

[1] Negishi, E.-I.; de Meijere, A. Handbook of Organopalladium Chemistry for Organic Synthesis, Wiley-Interscience, New York, 2002.

[2] For selected reviews on oxidative coupling reaction, see:

(a) Liu, C.; Zhang, H.; Shi, W.; Lei, A. Chem. Rev. 2011, 111, 1780.

(b) Shi, W.; Liu, C.; Lei, A. Chem. Soc. Rev. 2011, 40, 2761.

(c) Liu, C.; Yuan, J.; Gao, M.; Tang, S.; Li, W.; Shi, R.; Lei, A. Chem. Rev. 2015, 115, 12138.

(d) Zhang, J.; Lu, Q.; Li, C.; Lei, A. Chin. J. Org. Chem. 2015, 35, 743 (in Chinese).

(张剑，陆庆全，刘超，雷爱文，有机化学，2015, 35, 743.)

(e) Yi, H.; Zhang, G.; Wang, H.; Huang, Z.; Wang, J.; Singh, A. K.; Lei, A. Chem. Rev. 2017, 13, 9016. (f) Yuan, Y.; Lei, A. Acc. Chem. Res. 2019, 52, 3309.

[3] Kozlowski, M. C. Acc. Chem. Res. 2017, 50, 638.

[4] Murarka, S.; Antonchick, A. P. Synthesis 2018, 50, 2150.

[5] Ivanoff, D.; Spassoff, A. Bull. Soc. Chim. Fr. 1935, 2, 76.

[6] (a) Fujii, T.; Hirao, T.; Ohshiro, Y. Tetrahedron Lett. 1992, 33, 5823.

(b) Kohno, Y.; Narasaka, K. Bull. Chem. Soc. Jpn. 1995, 68, 322. (c) Ryter, K.; Livinghouse, T. J. Am. Chem. Soc. 1998, 120, 2658.

(d) Ekebergh, A.; Karlsson, I.; Mete, R.; Pan, Y.; Börje, A.; Mårtensson, J. Org. Lett. 2011, 13, 4458.

(e) Ding, H.; Roberts, A. G.; Harran, P. G. Angew. Chem., Int. Ed. 2012, 51, 4340.

(f) Tisovský, P.; Mečiarová, M.; Šebesta, R. Org. Biomol. Chem. 2014, 12, 9446.

(g) Vega, M. M.; Crain, D. M.; Konkol, L. C.; Thomson, R. J. Tetrahedron Lett. 2015, 56, 3228.

(h) Parida, K. N; Pathe, G. K.; Maksymenko, S.; Szpilman, A. M. Beilstein J. Org. Chem. 2018, 14, 992.

[7] (a) Rathke, M. W.; Lindert, A. J. Am. Chem. Soc. 1971, 93, 4605. (b) Dessau, R. M.; Heiba, E. I. J. Org. Chem. 1974, 39, 3457.

(c) Brocksom, T. J.; Petragnani, N.; Rodrigues, R.; LaScala Teixeira, H. Synthesis 1975, 396.

(d) Frazier, R. H., Jr.; Harlow, R. L. J. Org. Chem. 1980, 45, 5408. (e) Ojima, I.; Brandstadter, S. M.; Donavan, R. J. Chem. Lett. 1992, 1591.

(f) Paquette, L. A.; Bzowej, E. I.; Branan, B.; Stanton, K. J. J. Org. Chem. 1995, 60, 7277.

(g) Matsumura, Y.; Nishimura, M.; Hiu, H. J. Org. Chem. 1996, 61, 2809.

(h) McNulty, J.; Millar, M. J. J. Org. Chem. 1999, 64, 5312.

[8] (a) Kobayashi, Y.; Taguchi, T.; Tokuno, E. Tetrahedron Lett. 1977, 42,3741 .

(b) Mazzega, M.; Fabris, F.; Cossu, S.; De Lucchi, O. Tetrahedron 
1999, 55, 4427.

(c) Xie, J.; Huang, Z.-Z. Chem. Commun. 2010, 46, 1947.

(d) Kaiser, D.; Teskey, C. J.; Alder, P.; Maulide, N. J. Am. Chem. Soc. 2017, 139, 16040.

[9] Renaud, P.; Fox, M. A. J. Org. Chem. 1988, 53, 3745.

[10] Kise, N.; Tokioka, K.; Aoyama, Y.; Matsumura, Y. J. Org. Chem. 1995, 60, 1100.

[11] (a) Baran, P. S.; Richter, J. M.; Lin, D. W. Angew. Chem., Int. Ed. $\mathbf{2 0 0 5}, 44,609$

(b) Richter, J. M.; Whitefield, B. W.; Maimone, T, J.; Lin, D. W.; Castroviejo, M. P.; Baran, P. S. J. Am. Chem. Soc. 2007, 129, 12857.

(c) DeMartino, M. P.; Chen, K.; Baran, P. S. J. Am. Chem. Soc. 2008, 130, 11546.

[12] Csákÿ, A. G.; Plumet, J. Chem. Soc. Rev. 2001, 30, 313.

[13] Baran, P. S.; Ambhaikar, N. B.; Guerrero, C. A.; Hanfensteiner, B. D.; Lin, D. W. Richter, J. M. ARKIVOC 2006, (vii), 310.

[14] Dong, V. M.; Yeung, C. S. Chem. Rev. 2011, 111, 1215.

[15] Guo, F. H.; Clift, M. D.; Thomson, R. J. Eur. J. Org. Chem. 2012, 4881.

[16] Nagaraju, K.; Ma, D. W. Chem. Soc. Rev. 2018, 47, 8018.

[17] Xie, W. Q.; Zuo, Z. W.; Zi, W. W.; Ma, D. W. Chin. J. Org. Chem. 2013, 33, 869 (in Chinese). (谢卫青, 左智伟, 资伟伟, 马大为, 有机化学, 2013, 33, 869.)

[18] (a) Ito, Y.; Konoike, T.; Saegusa, T. J. Am. Chem. Soc. 1975, 97, 2912.

(b) Tokuda, M.; Shigei, T.; Itoh, M. Chem. Lett. 1975, 621.

(c) Ito, Y.; Konoike, T.; Harada, T.; Saegusa, T. J. Am. Chem. Soc. 1977, 99, 1487.

[19] Baran, P. S.; DeMartino, M. P. Angew. Chem., Int. Ed. 2006, 45, 7083.

[20] Casey, B. M.; Flowers, R. A. J. Am. Chem. Soc. 2011, 133, 11492.

[21] Nguyen, P. Q.; Schäfer, H. J. Org. Lett. 2001, 3, 2993.

[22] Konkol, L. C.; Guo, F. H.; Sarjeant, A. A.; Thomson, R. J. Angew. Chem., Int. Ed. 2011, 50, 9931.

[23] Mambrini, A.; Gori, D.; Guillot, R.; Kouklovsky, C.; Alezra, V. Chem. Commun. 2018, 54, 12742.

[24] Do, H. Q.; Hung, T. V.; Daugulis, O. Organometallics 2012, 31, 7816.

[25] Tsang, A.; Kapat, A.; Schoenebeck, F. J. Am. Chem. Soc. 2016, 138 , 518.

[26] Shang, M.; Sun, S.-Z.; Dai, H.-X.; Yu, J.-Q. Org. Lett. 2014, 16, 5666.

[27] Mao, S.; Gao, Y.-R.; Zhang, S.-L.; Guo, D.-D.; Wang, Y.-Q. Eur. J. Org. Chem. 2015, 876.

[28] Mao, S.; Zhu, X.-Q.; Gao, Y.-R.; Guo, D.-D.; Wang, Y.-Q. Chem.Eur. J. 2015, 21, 11335 .

[29] Geibel, I.; Christoffers, J. Eur. J. Org. Chem. 2016, 918.

[30] Tanaka, T.; Tanaka, T. Tsuji, T.; Yazaki, R.; Ohshima, T. Org. Lett. 2018, 20, 3541.

[31] For selected reviews on organic electrosynthesis, see: (a) Degner, D. In Electrochemistry III, Ed.: Steckchan, E., Springer, Berlin, 1988, pp. 1 95.

(b) Yoshida, J.; Kataoka, K.; Horcajada, R.; Nagaki, A. Chem. Rev. 2008, 108, 2265.

(c) Yan, M.; Kawamata, Y.; Baran, P. S. Chem. Rev. 2017, 117, 13230.

(d) Moeller, K. D. Chem. Rev. 2018, 118, 4817

(e) Wang, X. Y.; Xu, X. T.; Wag, Z. H.; Fang, P.; Mei, T. S. Chin. J. Org. Chem. 2020, 40, 3738 (in Chinese).

(王向阳, 徐学涛, 王振华, 方萍, 梅天胜, 有机化学, 2020, 40, 3738.)

[32] Huang, X. Q.; Zhang, Q.; Lin, J. H.; Harms K.; Meggers, E. Nat. Catal. 2019, 2, 34.

[33] Baciocchi, E.; Casu, A.; Ruzziconi, R. Tetrahedron Lett. 1989, 30, 3707.

[34] Schmittel, M.; Burghart, A.; Malisch, W.; Reising, J.; Söllner, R. J. Org. Chem. 1998, 63, 396.
[35] (a) Schmittel, M.; Söllner, R. Chem. Ber 1997, 130, 771.

(b) Schmittel, M.; Haeuseler, A. J. Organomet. Chem. 2002, 661, 169.

[36] Clift, M. D.; Taylor, C. N.; Thomson, R. J. Org. Lett. 2007, 9, 4667.

[37] Avetta, C. T.; Konkol, L. C.; Taylor, C. N.; Dugan, K. C.; Stern, C. L.; Thomson, R. J. Org. Lett. 2008, 10, 5621.

[38] Clift, M. D.; Thomson, R. J. J. Am. Chem. Soc. 2009, 131, 14579.

[39] Chen, W.; Guo, R. Y.; Gong, J. X.; Yang, Z. Chin. J. Org. Chem. 2019, 39, 238 (in Chinese). (陈伟，郭人予，龚建贤，杨震，有机化学， 2019, 39, 238.)

[40] Strehl, J.; Hilt, G. Org. Lett. 2019, 21, 5259.

[41] Narasaka, K.; Okauchi, T.; Tanaka, K.; Murakami, M. Chem. Lett. 1992, 2099

[42] Jang, H. Y.; Hong, J. B.; MacMillan, D. W. C. J. Am. Chem. Soc. 2007, 129, 7004.

[43] For selected reviews on photochemical synthesis, see:

(a) Romero, N. A.; Nicewicz, D. A. Chem. Rev. 2016, 116, 10075.

(b) Skubi, K. L.; Blum, T. R.; Yoon, T. P. Chem. Rev. 2016, 116, 10035 .

(c) Gentry, E. C.; Knowles, R. R. Acc. Chem. Res. 2016, 49, 1546.

(d) Twilton,J.; Le, C.; Zhang, P.; Shaw, M. H.; Evans, R. W.; MacMillan, D. W. C. Nat. Rev. Chem. 2017, 1, 0052.

(e) Ding, K.; Xiao, W.; Wu, L.-Z. Acta Chim. Sinica 2017, 75, 5 (in Chinese).

(丁奎岭, 肖文精, 吴骊珠, 化学学报, 2017, 75, 5.)

(f) Guan, B.; Xu, X.; Wang, H.; Li, X. Chin. J. Org. Chem. 2016, 36, 1564 (in Chinese).

(关保川, 许孝良, 王红, 李小年, 有机化学, 2016, 36, 1564.)

(g) Zhang, H. H.; Yu, S. Y. Chin. J. Org. Chem. 2019, 77, 832 (in Chinese).

(张洪浩, 俞寿云, 有机化学, 2019, 77, 832.)

(h) Li, Z. L.; Jin, J.; Huang, S. H. Chin. J. Org. Chem. 2020, 40, 563 (in Chinese).

(李祯龙，金健，黄莎华，有机化学, 2020,40, 563.)

[44] Yasu, Y.; Koike, T.; Akita, M. Chem. Commun. 2012, 48, 5355.

[45] (a) Li, Q.; Fan, A.; Lu, Z.; Cui, Y.; Lin, W.; Jia, Y. Org. Lett. 2010, 12,4066 .

(b) Li, Q.; Jiang, J.; Fan, A.; Cui, Y.; Jia, Y. Org. Lett. 2011, 13, 312.

[46] Gao, X. L.; Wang, P.; Wang, Q. Q.; Chen, J. T.; Lei, A. W. Green Chem. 2019, 21, 4941.

[47] Næsborg, L.; Corti, V.; Leth, L. A.; Poulsen, P. H.; Jørgensen. A. Angew. Chem., Int. Ed. 2018, 57, 1606.

[48] Amaya, T.; Masuda, T.; Maegawa, Y.; Hirao, T. Chem. Commun. 2014, 50, 2279.

[49] Amaya, T.; Maegawa, Y.; Masuda, T.; Osafune, Y.; Hirao, T. J. Am. Chem. Soc. 2015, 137, 10072.

[50] Amaya, T.; Osafune, Y.; Maegawa, Y.; Hirao, T. Chem. Asian J. 2017, 12, 1301.

[51] Osafune, Y.; Jin, Y. Q.; Hirao, T.; Tobisu, M.; Amaya, T. Chem. Commun. 2020, 56, 11697.

[52] Douglas, F. F.; Belletire, J. L. J. Org. Chem. 1988, 53, 4724.

[53] Quesnelle, C. A.; Gilla, P.; Kima, S.-H.; Chen, L. B.; Zhao, Y. F.; Flnk, B. E.; Saulnier, M.; Frennesson, D.; DeMartino, M. P.; Baran, P. S.; Gavai, A.V. Synlett 2016, 27, 2254.

[54] Richter, J. M.; Ishihara, Y.; Masuda, T.; Whitefield, B. W.; Llamas, T.; Pohjakallio, A.; Baran, P. S. J. Am. Chem. Soc. 2008, 130, 17938.

[55] Lu, H. L.; Zhu, G. R.; Tang, T. G.; Ma, Z.; Chen, Q.; Chen, Z, L. iScience 2019, 22, 214

[56] Krawczuk, P. J.; Schöne, N.; Baran, P. S. Org. Lett. 2009, 11, 4774.

[57] He, H.; Ding, W. D.; Bernan, V. S.; Richardson, A. D.; Ireland, C. M.; Greenstein, M.; Ellestad, G. A.; Carter, G. T. J. Am. Chem. Soc. 2001, 123, 5362 .

[58] Krygowski, E. S.; Murphy-Benenato, K.; Shair, M. D. Angew. Chem., Int. Ed. 2008, 47, 1680

[59] Lee, H. G.; Ahn, J. Y.; Lee, A. S.; Shair, M. D. Chem.-Eur. J. 2010, 16, 13058. 
[60] Herzon, S. B.; Lu, L.; Woo, C. M.; Gholap, S. L. J. Am. Chem. Soc. 2011, 133, 7260 .

[61] Ghosh, S.; Chaudhuri, S.; Bisai, A. Org. Lett. 2015, 17, 1373.

[62] You, L.; Liang, X.-T.; Xu, L.-M.; Wang, Y.-F.; Zhang, J.-J.; Su, Q.; Li, Y.-H.; Zhang, B.; Yang, S.-L.; Chen, J.-H.; Yang, Z. J. Am. Chem. Soc. 2015, 137, 10120.

[63] Cohen, T.; MaNamara, K.; Kuzenko, M. A.; Ramig, K.; Landi, Jr. J.; Dong, Y. Tetrahedron 1993, 49, 7931.

[64] (a) Baran, P. S.; Guerrero, C. A.; Ambhaikar, N. B.; Hafensteiner, B. D. Angew. Chem., Int. Ed. 2005, 44, 606.

(b) Baran, P. S.; Guerrero, C. A.; Hafensteiner, B. D.; Ambhaikar, N. A. Angew. Chem., Int. Ed. 2005, 44, 3892.

(c) Baran, P. S.; Hafensteiner, B. D.; Ambhaikar, N. B.; Guerrero, C. A.; Gallagher, J. D. J. Am. Chem. Soc. 2006, 128, 8678.

[65] (a)Martin, C. L.; Overman, L. E.; Rohde, J. M. J. Am. Chem. Soc. 2008, 130,7568 . (b) Martin, C. L.; Overman, L. E.; Rohde, J. M. J. Am. Chem. Soc. 2010, 132, 4894.

[66] (a) Zuo, Z.; Xie, W.; Ma, D. J. Am. Chem. Soc. 2010, 132, 13226

(b) Zuo, Z.; Ma, D. Angew. Chem., Int. Ed. 2011, 50, 12008.

(c) Zi, W.; Xie, W.; Ma, D. J. Am. Chem. Soc. 2012, 134, 9126.

(d) Wei, Y.; Zhao, D.; Ma, D. Angew. Chem., Int. Ed. 2013, 52, 129881

(e) Teng, M.; Zi, W.; Ma, D. Angew. Chem., Int. Ed. 2014, 53, 1814.

[67] Yang, H.; Feng, J.; Tang, Y. Chem. Commun. 2013, 49, 6442.

[68] Watanabe, T.; Kato, N.; Umezawa, N.; Higuchi, T. Chem.-Eur. J. 2013, 19, 4255.

[69] Du, J. N.; Espelt, L. R.; Guzei, I. A.; Yoon, T. P. Chem. Sci. 2011, 2 , 2115.

[70] Robison, E. E.; Thomson, R. J. J. Am. Chem. Soc. 2018, 140, 1956.

[71] Jones, B. T.; Avetta, C. T.; Thomson, R. J. Chem. Sci. 2014, 5, 1794. 